\title{
Wave-particle interactions associated with nongyrotropic distribution functions: A hybrid simulation study
}

\author{
P. D. Convery, ${ }^{1}$ D. Schriver, ${ }^{2}$ M. Ashour-Abdalla, ${ }^{1,2}$ and R. L. Richard ${ }^{2}$ \\ Received 7 June 2001: revised 13 August 2001; accepted 17 August 2001: published 23 January 2002.
}

[1] Nongyrotropic plasma distribution functions can be formed in regions of space where guiding center motion breaks down as a result of strongly curved and weak ambient magnetic fields. Such are the conditions near the current sheet in the Earth's middle and distant magnetotail, where observations of nongyrotropic ion distributions have been made [Frank et al., 1994]. Here a systematic parameter study of nongyrotropic proton distributions using electromagnetic hybrid simulations is made. We model the observed nongyrotropic distributions by removing a number of arc length segments from a cold ring distribution and find significant differences with the results of simulations that initially have a gyrotropic ring distribution. Model nongyrotropic distributions with initially small perpendicular thermalization produce growing fluctuations that diffuse the ions into a stable Maxwellian-like distribution within a few proton gyro periods. The growing waves produced by nongyrotropic distributions are similar to the electromagnetic proton cyclotron waves produced by a gyrotropic proton ring distribution in that they propagate parallel to the background magnetic field and occur at frequencies on the order of the proton gyrofrequency. The maximum energy of the fluctuating magnetic field increases as the initial proton distribution is made more nongyrotropic, that is, more highly bunched in perpendicular velocity space. This increase can be as much as twice the energy produced in the gyrotropic case. INDEX TERMS: 7867 Space Plasma Physics: Wave/particle interactions, 7843 Space Plasma Physics: Numerical simulation studies, 2772 Magnetospheric Physics: Plasma waves and instabilities, 2764 Magnetospheric Physics: Plasma sheet; KEYWORDS: Plasma instabilities, nongyrotropic distributions, hybrid simulations

\section{Introduction}

[2] The vast majority of studies concerning linear and nonlinear plasma instabilities in the magnetosphere involve particle distributions that are gyrotropic. Part of the reason for this is that in many regions of the magnetosphere, gyrotropic, Maxwellian-like distributions are commonly observed and provide an adequate description of the plasma. Recent observations and theory have led to an interest in understanding non-Maxwellian particle distribution functions that can be classified as nongyrotropic. A nongyrotropic plasma is characterized by a velocity distribution that satisfies

$$
\frac{\partial f\left(v_{3} \cdot v_{+} \cdot c\right)}{\partial \Phi} \neq 0
$$

where $f$ is the three-dimensional distribution function in cylindrical velocity coordinates, the velocity coordinates are given by $v_{l}=v_{x}$. $v=v_{1}^{2}+v_{2}^{2}$, and $\phi=\tan ^{-1}\left(v-v_{y}\right)$ is the gyrophase angle in the perpendicular velocity plane. The directions parallel and perpendicular are with respect to the background magnetic field (indicated by $\mathbf{B}_{\mathrm{o}}$ ).

[3] Recent observations by the Galileo and Geotail satellites in the Earth's magnetotail have established that ion distribution functions can have non-Maxwellian, nongyrotropic features [Frank et al. 1994; Saito et al., 1994]. In fact, the Galileo data led Frank et al. [1994] to conclude that distributions in the intermediate $\left(x \approx 35 R_{E}\right)$

\footnotetext{
${ }^{1}$ Department of Physics and Astronomy. University of California at Los Angeles, Los Angeles, California. USA.

Institute of Geophysics and Planetan Physics. University of Califomia at Los Angeles, Los Angeles, California. USA
}

Copyright 2002 by the American Geophysical Union. $0] 48-0227 / 02 / 2001$ J A000 I $60 \$ 09.00$ and distant magnetotail cannot accurately be described in terms of convecting, quasi-Maxwellian distributions.

[4] The Geotail spacecraft also observed ring-like proton velocity distributions that are somewhat nongyrotropic in the region between the plasma sheet and the lebe in the distant tail [Saito et al., 1994]. Cold proton beams observed in the lobe are believed to be the source of the nongyrotropic protons. These beams can be accelerated by thermoelectric fields associated with slow mode shocks. If the spatial scale of the electric field is smaller than the ion gyro radius, the ions can form a ringshaped distribution [Saito et al., 1994]. The perpendicular (to $B_{0}$ ) speeds are observed to be on the order of a few hundred kilometers per second (for reference, the Alfven speed is $\sim 2300$ $\mathrm{km} / \mathrm{s}$ ) and ring proton to total proton density ratios ranging from 0.20 to 1.00 . During a 1 -month period the Geotail spacecraft spent 80 hours in the area of the plasma sheet-lobe boundary During that time, 25 examples of ring-like distributions with varying degrees of nongyrotropicity were observed. Figure 1c of Saito et al. [1994] shows such a distribution.

[5] Large-scale modeling studies have also supported the idea that naturally occurring nongyrotropic-like distributions may be an important feature in the magnetosphere. For example, using largescale kinetic (LSK) calculations, Ashour-Abdalla et al. [1993a] showed that azimuthally nonsymmetric distributions can form in the central plasma sheet (CPS) that can increase the free energy to levels above those of the standard loss cone distributions. LSK results have also shown that Maxwellian ion distributions from the mantle can lead to the formation of a thin current sheet in which the ion pressure tensor has significant off-diagonal terms, indicative of the presence of nongyrotropic distributions [Ashour-Abdalla et al., 1994]. These nongyrotropic distributions formed by ion nonadiabatic motion in the magnetotail are in good qualitative agreement with the main features of the Galileo observations [Ashour-Abdalla et al., 1996]. Using a centrifugal impulse model, Delcourt and Martin [1994, 1999] also demonstrated that nonadiabatic particle 
motion in the magnetotail could lead to gyrophase bunching when the particles do not conserve their magnetic moment near the magnetotail midplane, where local magnetic field gradients are of the order of the Larmor radius. Particle trajectory studies have also shown that off-diagonal terms in the pressure tensor of the magnetotail are essential for maintaining the pressure balance and the tail structure of a thin current sheet at distances $\geq 20 R_{E}$ trom Earth [Ashour-Abdalla et al., 1993b]. These terms are a direct consequence of nongyrotropic distributions and are nonexistent for a gyrotropic plasma. Because of their lack of self-consistency, however, test particle and LSK methods cannot be used to investigate the effects of wave particie interactions or other collective effects that could be caused by nongyrotropic distributions.

[6] The question that arises is whether the nongyrotropic nature of these distributions represents a significant source of free energy that can drive plasma instabilities. If so. this would imply that nongyrotropic aspects of a velocity distribution should be included in theoretical and simulation models applied to the magnetotail and other regions of space where nongyrotropic distributions may be found.

[7] The relevance of studies of nongyrotropic ion distributions is not limited to the magnetotail applications. Observations of nongyrotropic ions have been made by the Giotto spacecraft near the comet $\mathrm{P} / \mathrm{Grigg}$-Skjellerup (P/G-S), where ring-like densities, largest near the comet, have been observed up to several particles per cubic centimeter [Coates et al., 1993]. Perpendicular speeds are on the order of $400 \mathrm{~km} / \mathrm{s}$. In a study of low-frequency electromagnetic waves observed near $\mathrm{P} / \mathrm{G}-\mathrm{S}$, Neubauer et al. [1993] found waves with a peculiar character near to, but upstream of the cometary bow shock and suggested that the unusual characteristics may be due to either the nongyrotropic ions seen there or nonlinear effects. These waves are left-hand polarized, propagate along the background magnetic field (within $\pm 10^{\circ}$ ), have frequencies close to the local ion cyclotron frequency, and are observed in conjunction with the nongyrotropic ions. The unique aspect of these waves is that they are nearly sinusoidal with sharp peaks and rounded troughs [i.e., see Neubauer et al., 1993, Figures 10a and 10b]. They note, however, that "much more theoretical work is needed to assess the importance of nongyrotropy for the observed wave fields" (p. 20,950). More recently, Astudillo et al. [1996] used data from the PHOBOS Il mission to Mars to provide clear evidence of nongyrotropic alpha particles and protons in the solar wind at $\sim 1.3 \mathrm{AU}$.

[8] The existence of nongyrotropic ions in space plasmas is well established. While the quasi-linear and nonlinear consequences of these distributions have only recently been explored in the literature, studies concerning the linear stability of nongyrotropic ion distributions have been conducted for several years. Sudan [1965] was the first to derive the dispersion relation for parallel propagating, electromagnetic instabilities driven by a nongyrotropic plasma. Fredericks [1975] performed a linear stability analysis of Alfven waves driven by protons that had been partially gyrophase bunched by being reflected off the Earth's bow shock. In subsequent years, similar studies were conducted on the linear dispersion of parallel propagating electromagnetic waves driven by nongyrotropic ions [Brinca et al., 1992, 1993a] and electrons [Fruchtman and Fried[Brind 1983; Freund et al., 1987]. Linear theory was applied by Motschmann and Glassmeier [1993] to investigate the instabilities generated by nongyrotropic cometary ions, and Cao et al. [1995, 1998] studied the linear properties of model nongyrotropic ion distributions found near comet $\mathrm{P} / \mathrm{Grigg-Skjellerup.}$

[9] Brinca and Romeiras [1998] did a linear stability study of stationary nongyrotropic distributions, that is, those distributions that can be maintained by particle sources and sinks which can be used to model the plasma of cometary environments. This model differs from ours in that we will study simulations of homogeneous, time-varying nongyrotropic distributions with no sources or sinks. Brinca and Romeiras [1998] showed that adding a stationary nongyrotropic distribution to an otherwise stable plasma population could bring about linear instability in parallel eigenmodes. Brinca [1999] studied the linear stability of both stationary and time-varying nongyrotropic distributions and showed that their free energy can destabilize a plasma and enhance the growth of instabilities driven by gyrotropic distributions. The linear theory f both stationary and time-varying nongyrotropic distributions was aiso studied by Motschmann and Glassmeier [1998] and Motschmann et al. [1999] for a particular parameter regime. They showed that stationary nongyrotropic distributions drive both perpendicular and parallel propagating waves unstable, while time stationary nongyrotropic distributions led primarily to an instability of perpendicular waves. Burinskaya et al. [1994] investigated stability properties of electrostatic waves in the presence of ion distributions with velocity space holes. These holes are another way of modeling the nongyrotropic ions produced by chaotic ion acceleration in the neutral sheet.

10] Only a few studies have used simulations to investigate the nonlinear evolution of nongyrotropic ions. Gurgiolo and Wong [1993] found that shock-produced gyrophase bunched ions generated parallel propagating, low-frequency hydromagnetic and high-frequency whistler waves in a one-dimensional hybrid simulation. In some cases the low-frequency waves enhanced the gyrophase bunching through particle trapping, and in others they scattered the distribution toward gyrotropy.

[11] Brinca et al. [1993b] used a one-dimensional electromagnetic hybrid code to compare instability growth driven by anisotropic, gyrotropic protons versus growth by anisotropic, nongyrotropic protons. They made the protons severely nongyrotropic by restricting their initial gyrophase angles to $0= \pm \pi / 2$. The growth of the fluctuating magnetic field energy density was found to occur faster for the nongyrotropic case; however, the saturation level of the magnetic energy was about the same. The investigators interpreted this to mean that both the gyrotropic and nongyrotropic cases were driven by the same free energy, that is, the temperature anisotropy. Another difference between the two cases was the magnetic energy, which tended to oscillate in the nongyrotropic case as energy was exchanged between the gyrophase bunched protons and the electromagnetic fields. Last, they found that the nongyrotropic nature of the protons persisted until several gyro periods after saturation.

12] Motschmann et al. [1997] used a two-dimensional hybrid code to study nongytotropic ions in association with comets by injecting spatially homogeneous alpha particles perpendicular to the background magnetic field for a time shorter than an ion gyto period. This process produced nongyrotropic distributions that in velocity space look like rings with two arc lengths removed. The main findings concemed two types of velocity diffusion. Radial diffusion occurred on timescales on the order of $10 \Omega_{p}{ }^{-1}$, where $\Omega_{p}$ is the proton gyrofrequency. Diffusion in 作 the azimuthal direction depended on the ring distribution and could be as fast as radial diffusion. There was no discussion of saturation processes. Recently, Cao et al. [2000] used a one-dimensional electromagnetic hybrid code to study the pickup process and diffusion of newbom ions in solar wind plasmas. The parallel propagating electromagnetic ion cyclotron ring instability dominated and led both phase and pitch angle diffusion on a timescale of the order of a few proton gyro periods. Phase angle scattering of the ions occurred slightly faster than pitch angle scattering and much faster than energy diffusion.

[13] Brinca et al. [1998] considered one-dimensional electro[13] Brinca el al. [19tic in cell simulations of timestatic and electromagnetic pistribution functions. They found that vary electrostatic cyclotron harmonic waves mode. The perpendicular as well as the electromagnetic ordinary mode. The perpendicular instabilities resulted in gyroph 
[14] The magnetospheric observations highlight the need for a thorough study of nongyrotropic distributions and their nonlinear consequences in order to determine their role in magnetospheric physics. The importance of nongyrotropic distributions as a source of free energy to drive instabilities is still not clear, nor is it clear if the gyrotropic plasma particle picture of the magnetotail used in many theoretical studies needs to be modified to include these effects. This paper will address these issues by carrying out a systematic parametric simulation study of nongyrotropic distribution functions. The goal is to contribute to the understanding of the consequences of nongyrotropic distributions and help form a more complete picture of the magnetotail.

[15] In section 2 the hybrid simulation code used for all of the simulations in this paper is described. We also discuss in more detail the observational foundations for the model nongyrotropic distribution used in this study. Section 3 includes a detailed analysis of a simulation using a ring distribution and compares these results to several other simulations using different types of nongyrotropic distributions. Time histories of total field energy. velocity space diffusion, and individual electric field and power spectrum characteristics are discussed. This section also presents results of the growing fluctuating field dependence on the initial values of the perpendicular beam speed, the nongyrotropic proton distribution density, and the shape of the nongyrotropic distribution in velocity space. A detailed discussion of the saturation mechanism involved in the growing waves is also presented in this section. Finally, properties of model nongyrotropic distributions that may cause the plasma to maintain stability even though it is highly non-Maxwellian are presented. Section 4 summarizes our conclusions and discusses the relevance of these results to local and global magnetospheric physics.

\section{Hybrid Code and Model Distribution}

[16] The simulations presented in this study are carried out using a two-dimensional electromagnetic hybrid code. This code was originally written by Dan Winske and is based on his onedimensional code described by Winske and Omidi [1993]. The main advantage of a hybrid code is the great economy of computing time achieved by treating the electrons as a massless fluid. A fully kinetic description of protons is used, so the full range of ion spatial and temporal scales is resolved. The code also sets the displacement current equal to zero in Maxwell's equations. Such a scheme is valid for the study of low-frequency electromagnetic processes such as those that we are interested in. The simulation box is periodic in the two spatial dimensions $(x-y)$. each having system length $L_{x}=L_{y}=32 c / \omega_{p}$, where $c$ is the speed of light and $\omega_{\mathrm{p}} \equiv 4 \pi n_{\mathrm{p}} q^{2} / m_{\mathrm{p}}$ is the total proton plasma frequency. The system length was chosen so that it adequately resolves the dominant growing modes as determined by the linear theory dispersion relation for a proton ring distribution [Convery and Gan; 1997].

[17] Fluctuating fields are allowed to propagate freely in the $(x-$ y) plane. There is a uniform ambient magnetic field in the $x$ direction. The simulation is carried out in the reference frame where the electrons are at rest. Complete simulation parameters are listed in Table 1.

[18] All protons are loaded uniformly in space. and the background protons (if any) have density $n_{\text {back }}$ and are given by a threedimensional, non-drifting Maxwellian velocity distribution. The total proton density $n_{\mathrm{p}}$ satisfies $n_{\mathrm{p}}=n_{\mathrm{ng}}+n_{\mathrm{beck}}=n_{\mathrm{e}}$. where $n_{\mathrm{ng}}$ is the nongyrotropic proton density and $n_{c}$ is the total electron density. The nongyrotropic proton distribution is Maxwellian in the parallel direction and is modeled by a cold ring in the perpendicular $\left(v_{y}-v_{z}\right)$ velocity plane. The ring distribution is then modified by removing one or more arc length segments of various sizes to create a nongyrotropic distribution. The total azimuthal width of these sections determines the nongyrotropic
Table 1. Range of Parameters Used for the Hybrid Simulations, Unless Otherwise Noted in the Text

\begin{tabular}{|c|c|}
\hline Parameter & Value \\
\hline $\begin{array}{l}\text { System length } \\
\text { Grid size } \\
\text { Time step } \\
\text { Total nun time } \\
\text { Total number of particles } \\
\text { Nongyrotropic plasma density } \\
\text { Perpendicular velocity } \\
\text { Electron beta } \beta_{c} \\
\text { Initial proton beta } \beta_{i, p} \\
\end{array}$ & $\begin{array}{l}L_{x}=L_{\mathrm{y}}=32 c / \nu_{\mathrm{p}} \\
\Delta x=\Delta y=c / u_{\mathrm{pl}} \\
\Delta t=0.05 \Omega_{\mathrm{p}}-1 \\
25 \leq \Omega_{\mathrm{p}} \leq 100 \\
819,200 \leq N \leq 1.310 .720 \\
0.01 \leq n_{\mathrm{ng}} / n_{\mathrm{c}} \leq 1.0 \\
1.0 \leq v_{\perp 0} / v_{\mathrm{A}} \leq 5.0 \\
1.0 \\
10^{-4} \leq \beta_{\mathrm{p}} \leq 1.0\end{array}$ \\
\hline
\end{tabular}

nature of the distribution at any given time. In section 3.1 a parameter $\Phi$ is defined that quantifies the nongyrotropic aspect for any given distribution. The parallel proton plasma beta $\beta_{\text {kp }} \equiv$ $8 \pi n_{\mathrm{e}} T_{1 \mathrm{p}} / \mathrm{B}_{\mathrm{o}}{ }^{2}=10^{-4}$, unless otherwise noted. The Alfvén speed is $v_{\mathrm{A}}=\mathrm{B}_{\mathrm{o}}{ }^{2} / 4 \pi n_{\mathrm{c}} m_{\mathrm{p}}$, and we set $v_{\mathrm{A}} / c=10^{-4}$. The electron temperature is fixed and is given by $3_{\mathrm{e}} \equiv 8 \pi n_{\mathrm{e}} T_{\mathrm{e}} / \mathrm{B}_{\mathrm{o}}{ }^{2}=1.0$.

[19] Our nongyrotropic model is chosen foremost because it closely resembles the observations of nongyrotropic particles in the Earth's magnetotail [e.g., Saito et al., 1994]. We also benefit from the added advantage that the simulation results using this model can be compared to results from simulations using the wellunderstood ring distribution. This proves to be very helpful in interpreting the simulation results and in isolating uniquely nongyrotropic effects. Both to check our work and to provide insight into the physics, we contrast our nongyrotropic results with the ring case results throughout this paper.

[20] Although the linear theory for a nongyrotropic distribution is not derived here, it is worth discussing the validity of a model nongyrotropic distribution in terms of linear Vlasov theory in anticipation of future linear theory investigations of nongyrotropic distributions. A nongyrotropic distribution function means that $\partial f \partial \phi \neq 0$, where $\phi$ is the azimuthal angle in the velocity plane perpendicular to the ambient magnetic field. Therefore, in order to satisfy the Vlasov equation with no source or sink terms, the equilibrium nongyrotropic distribution must also be either time dependent $(\partial f / \partial t \neq 0)$ or spatially inhomogeneous $(\partial f / \partial \mathbf{x} \neq 0)$ Sudan, 1965], where $\mathbf{x}$ is the three-dimensional position vector. It is important to note that in a plasma environment with local particle sources and sinks, an equilibrium nongyrotropic distribution that is both time stationary and homogeneous can be a solution to the Vlasov equation in an open phase space. Although studied here, such a model is appropriate when the plasma population includes pickup ions, such as in cometary environtsee references in section 1). For the plasma environment mere (no particle sources or sinks), this implies that a nongyrotropic distribution must have a functional dependence given by $f_{\mathrm{ng}}^{\circ}=f_{\mathrm{ng}}{ }^{\circ}\left(v_{1}, v_{1}, 0-\Omega t\right)$ for the time-dependent case or for the spatially dependent case $f_{\mathrm{ng}}{ }^{\circ}=f_{\mathrm{ng}}{ }^{\circ}\left(v_{1,}, v_{\perp} .0-\Omega \mathbf{x} / v_{\|}\right)$. Our model, bunched in velocity space and spatially uniform, is an example of the time-dependent form and is therefore a Vlasov solution. Although not discussed here, an example of nongyrotropic particle distributions showing spatial bunching was given by Eastman et al. [1981], who observed ion distributions in the foreshock region displaying gyrophase organization with a spatial scale less than an ion gyro radius

[21] Motschmann et al [1997] have done a simulation study with a two-dimensional hybrid code using a nongyrotropic model somewhat similar to what is used here. The primary differences with this work are that their work focused on parameters relevant to cometary environments. specifically, comet P/Grigg-Skjellerup. where the nongyrotropic ions are alpha particles and the ratio of their density to total ion density is of the order of a few percent. We emphasize parameters associated with the Earth's magnetotail. where nongyrotropic proton densities can be as much as $100 \%$ 


\section{Examples of $\Phi_{0}$}
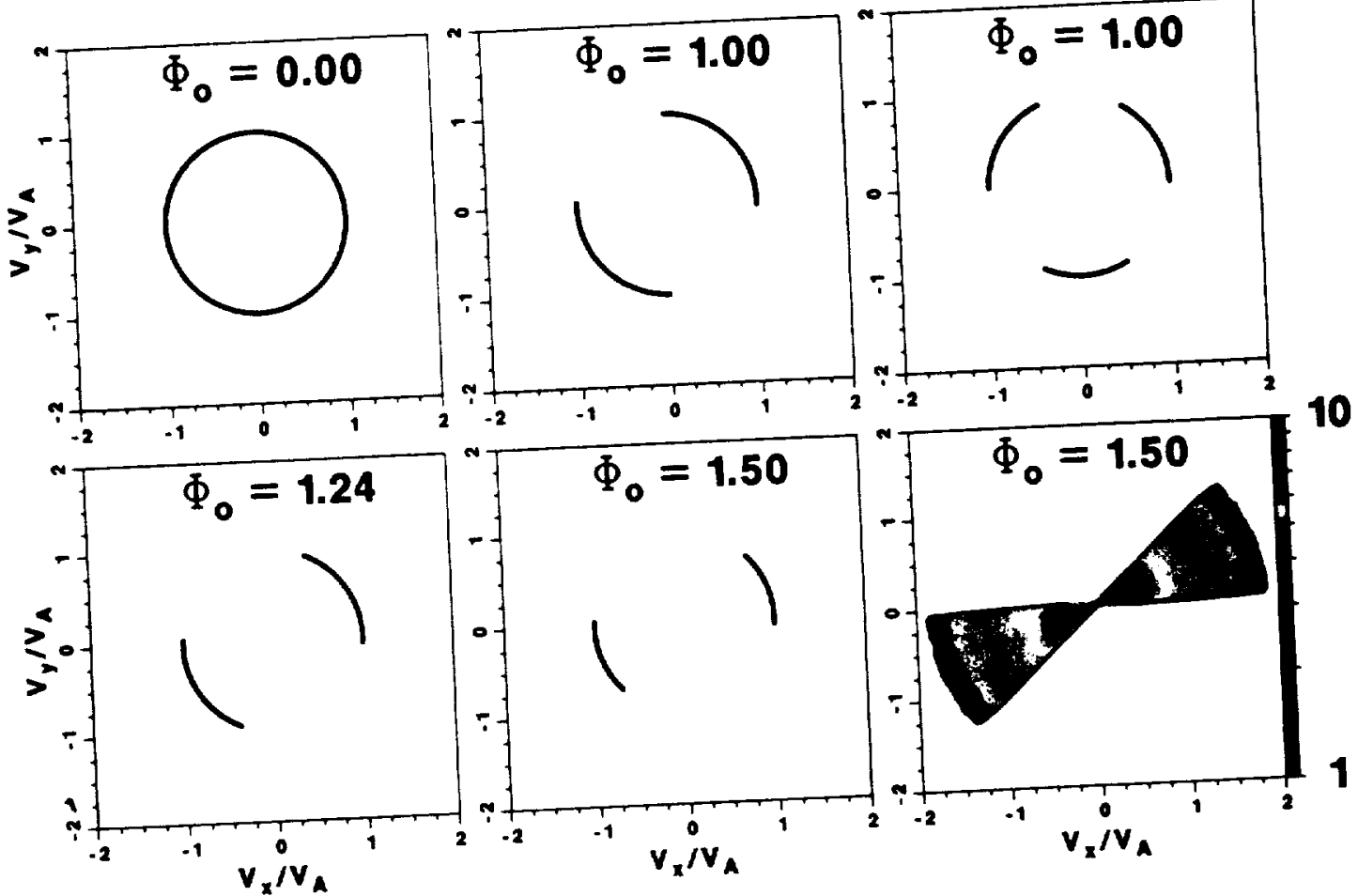
Figure 1. Velocity distribution in the plane perpendicular to the ambient
of the parameter $\Phi_{0}$. A gyrotropic ring distribution corresponds to $\Phi_{0}=0$.

of the observed proton density. Another significant difference is that our interpretation of oults of simulations using nonthat our intions is facilitated through a comparison gyrotropic proton distributions is facilitated throughtion. No such to results from simulations using a ting distribs

\section{Results of Hybrid Simulations}

\subsection{Definition of Parameters}

22] Both the ring and nongyrotropic proton distributions studied here are unstable to electromagnetic ion cyclotron waves for certain magnetotail parameters. We can quantify the particle response to the flucruating fields in terms of the following four parameters:

Parallel proton beta

$$
3_{i \mathrm{p}} \equiv \frac{8 \pi n_{\mathrm{e}} T_{\mathrm{p}}}{B_{\mathrm{o}}^{2}}=\frac{\left.2\left(<v_{i}^{2}>-<v_{i}\right\rangle^{2}\right)}{\nu_{i}^{2}} .
$$

Average normalized perpendicular ring speed squared

$$
\left\langle v^{2}\right\rangle / v_{A}^{2}
$$

A perpendicular thermal factor

$$
b_{i g} \equiv \frac{\left.\left.2\left(<v_{-}^{2}\right\rangle-<v_{-}\right\rangle^{2}\right)}{v_{i}^{2}} .
$$

A parameter characterizing the nongyrotropic nature of the distribution

$$
\Phi(t) \equiv \int_{0}^{2 \pi}\left|\frac{n_{\mathrm{ng}}(\Phi, t)}{n_{\mathrm{o}}}-1\right| d \phi .
$$

In this last expression, $n_{\mathrm{ng}}(\phi, t)$ is the density per unit angle of the ic protons at a given azimuthal angle $\Phi$ and time $t$, and $n_{\text {ng }}$ at $t=0$ satisfies

$$
n_{0}=\frac{1}{2 \pi} \int_{0}^{2 \pi} n_{\mathrm{ng}}(\phi . t=0) d \phi .
$$

With this definition, $\Phi(t)$ quantifies the azimuthal diffusion of the proton distribution with time. The notation $\Phi_{0}$ is used throughout this paper to mean $\Phi(t=0)$. Examples of distributions with different $\Phi_{0}$ are shown in Figure 1. The top left panel in Figure 1 is a gyrotropic ring distribution $\left(\Phi_{0}=0\right)$. The top middle panel is a nongyrotropic distribution that has two diametrically opposed bunches of particles and $\Phi_{0}=1.0$. The top right panel also has $\Phi_{0}=1.0$, except it has three bunches of particles. This highlights the fact that $\Phi_{0}$ is not unique and does not take into account bunch number or spacing in the azimuthal direction, that is, it is only a function of the total azimuthal spread of the distribution. Any differences in the simulation results produced by these two exare work to inctud imply that $\Phi_{0}$ should be modified in future work that car other characteristics (bunch number and spactom left pane distinguish nongyrotropic distributions. The bottom left pottor shows two-bunch distribution with $\Phi_{0}=1.24$. The boun 

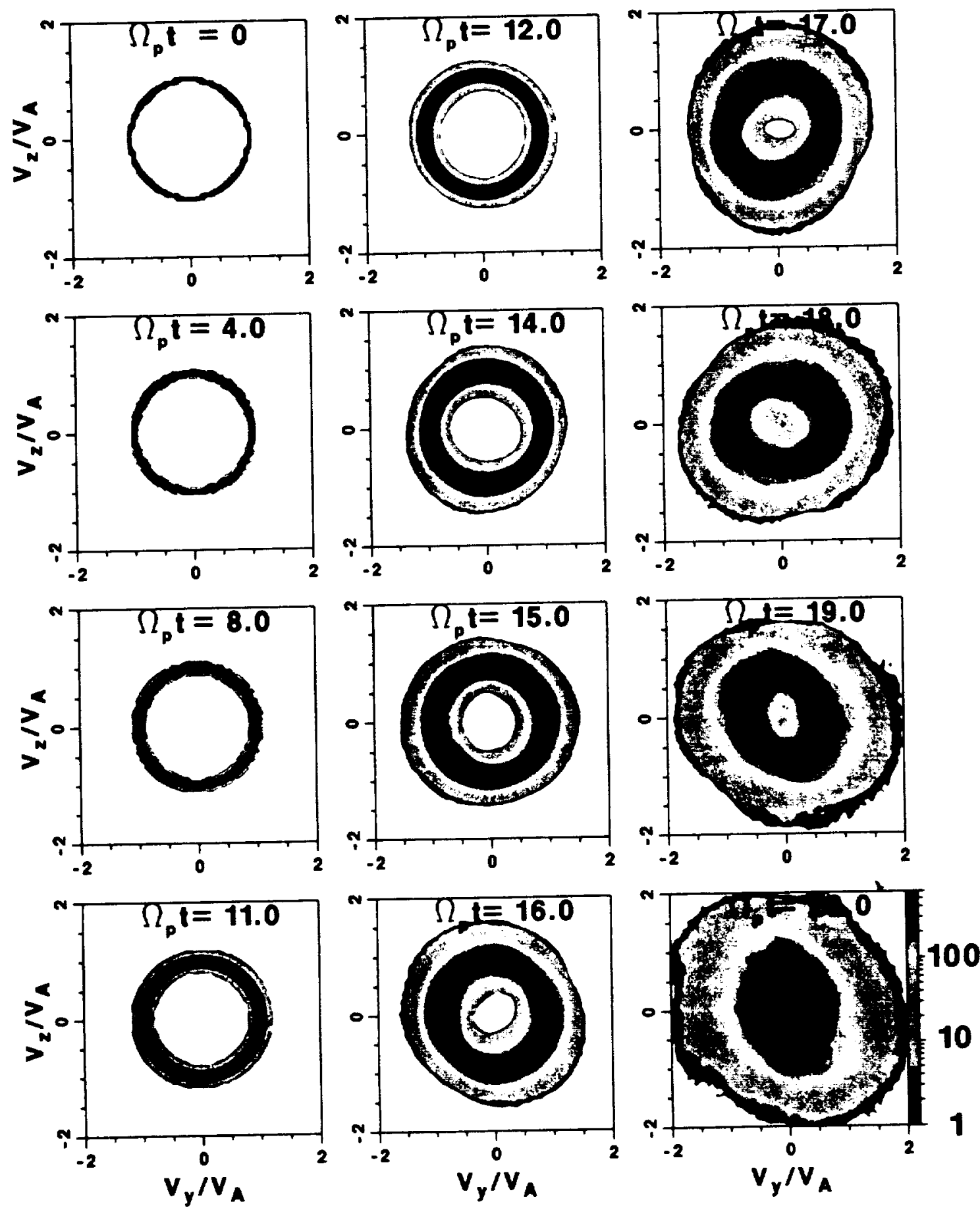

Figure 2. Velocity space time series in the plane perpendicular to the background magnetic field for an initially cold Fros radial direction and by $\Omega_{\mathrm{p}} t \approx 23$. the distribution has lost most of the positive gradient $\left(\partial f \partial_{-}>0\right)$ which drives the instability.

middle and bottom right panels both show distributions that have $\Phi_{0}=1.5$; however, the last distribution (bottom right panel) has a significant thermal spread in the perpendicular velocity. By comparing these last two cases, we seek to determine the effects of a nongyrotropic distribution with a perpendicular thermal spread. The thermal distribution is made perpendich it has no $\partial f \partial \partial>0$ slope. so if it were a gyrotropic distribution, it would be stable. We will determine whether or not making this thermal distribution nongyrotropic (i.e.. such that the distribution has $\partial f \partial o>0)$ by removing two large arc length segments will cause it to be unstable.

\subsection{Individual Runs}

3.2.1. Ring distribution. [23] To serve as reference for the nongyrotropic cases to follow: in Figure 2 we show a time series for an unstable proton ring distribution in the perpendicular velocity plane. For the rest of the paper this simulation nun will be referred to as the "ring case." For the nng case we use $v_{-\alpha} v_{A}=1.0$ and 

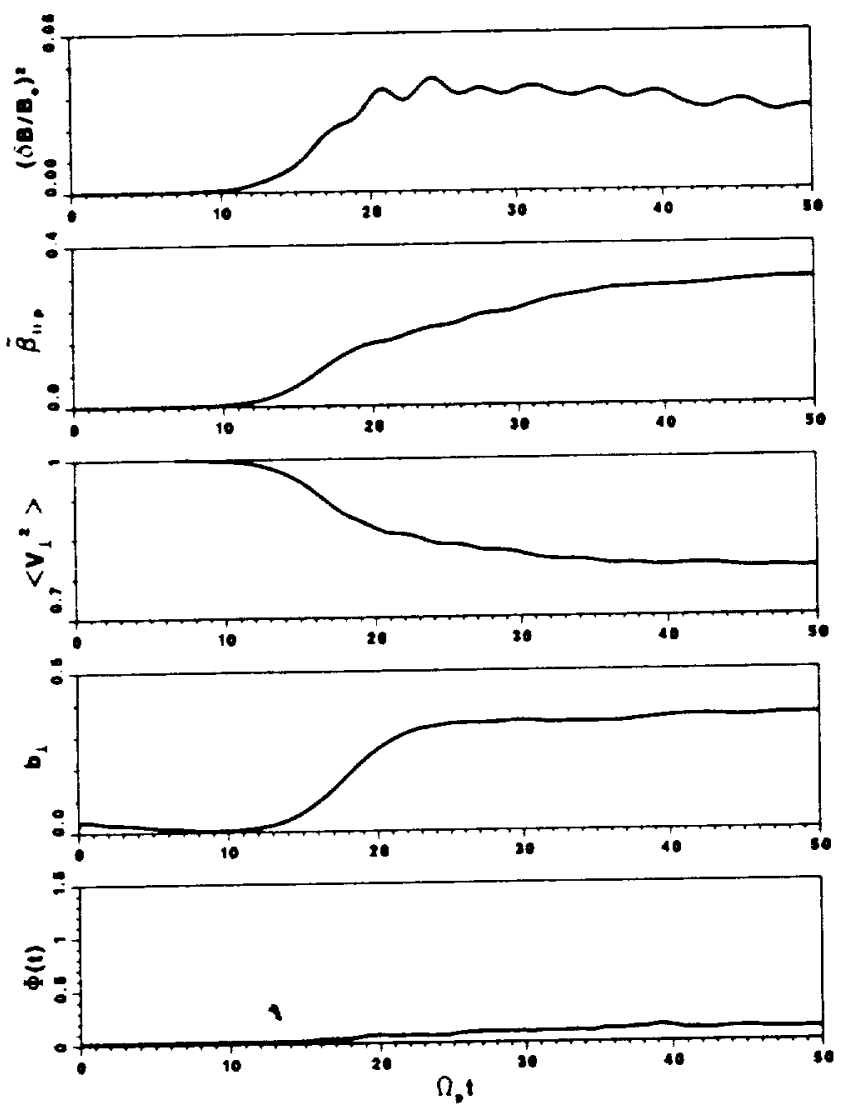

Figure 3. Time evolution of particle diagnostics for the distribution shown in Figure 2.

$n_{\text {ring }} / n_{\mathrm{e}}=1.0$. Figure 3 shows the corresponding time history for fluctuating field wave energy $\left(\delta B / B_{o}\right)^{2}$, and the four diagnostics defined above. Field saturation occurs at $\Omega_{\mathrm{p}} t \approx 20$ in conjunction with $\partial f / \partial v_{i} \rightarrow \sim 0$. Note the initial sharp increase in $3_{\| \mathrm{p}}$ and the sharp decrease in $\left\langle v_{\perp}{ }^{2}\right\rangle$ just before saturation. While $\beta_{\| p}$ continues to increase. $\left(v_{-}^{2}\right)$ continues to decrease, but at slower rates during the nonlinear stage. The sharp rise in $b_{i}$ stops just after saturation. By this time the ring protons have completely diffused to fill in the center of the ring, as seen in the bottom right panel of Figure 2 . The parameter $\Phi(t)$ starts at zero and remains at low levels throughout the run, as expected for a gyrotropic distribution.

3.2.2. Nongyrotropic distribution: Run 1. [24] Section 3.2.1 gave an overview of the effects produced by an unstable ring distribution. Here we begin with a similar analysis of several different nongyrotropic distributions. Figure 4 shows the perpendicular velocity space time series for a nongyrotropic distribution. This run uses $v_{\perp d} v_{\mathrm{A}}=1.0, n_{\mathrm{ng}} / n_{\mathrm{e}}=1.0$, and $\Phi_{\mathrm{o}}=$ 1.0. By $\Omega_{\mathrm{p}} t \approx 11$ the particles have diffused significantly in the radial direction, while azimuthal diffusion is minor, and the two gaps are still clearly visible. Figure 5 shows the time variation of the four quantities defined above as well as $\left(\delta B / B_{0}\right)^{2}$ for run 1 (gray) and for the ring case (black). The field energy saturates at $\Omega_{\mathrm{p}} t \approx 17$ with amplitude $\left(\delta B / B_{0}\right)^{2} \approx 0.04$. Oscillations in the fluctuating field energy at $\omega_{\mathrm{r}} \Omega_{\mathrm{p}} \approx 0.7$ are found (not shown) after saturation. The field energy of run $I$ peaks sooner and at a larger amplitude than the ring case, while the next three parameters are generally the same. $\Phi(t)$ shows some correlation with the field energy rise and saturation. At the first peak in $\left(\delta B / B_{0}\right)^{2}$ the distribution is still strongly nongyrotropic. By the second peak in the field energy, the distribution has become more gyrotropic, and the rapid diffusion in the radial direction has slowed. An interesting fearure to point out is the asymmetry in the radial diffusion. Figure 4 shows that as the protons undergo their Larmor gyromotion in the clockwise direction about the magnetic field, the leading edge of the distribution in the perpendicular velocity plane diffuses faster than the trailing edge. This may have something to do with the fact that in the rest frame of the particles, the circularly polarized fluctuating electric and magnetic fields seem to sweep by the particles in a counterclockwise direction because they rotate with frequency $\nu<\Omega_{\mathrm{p}}$. Consequently, the fields encounter the leading edge of the distribution first.

3.2.3. Nongyrotropic distribution: Run 2. [25] The distribution used for this run (not shown) is the same as in the previous case except the perpendicular velocity has been doubled to $v v_{\mathrm{A}}=2.0$. The main difference between the two runs is that

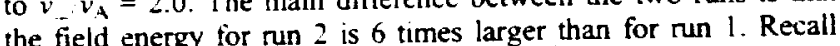
that the field energy is proportional to $v_{-}{ }^{2}$ for an unstable ring distribution. The results from the two runs and their comparisons to gyrotropic distributions are otherwise qualitatively the same. The main difference between these two cases is that the fields in run 2 show several small-amplitude spikes in the waveform while the ring simulation produced only a few. The spikes have frequencies greater than $\Omega_{\mathrm{p}}$. These characteristics were not seen in run 1 . implying that the purely nongyrotropic effects may only appear for distributions with sufficiently high perpendicular velocities.

3.2.4. Nongyrotropic distribution: Run 3. [26] The next run is motivated by observations that indicate that nongyrotropic distributions could be made of multiple as well as single bunches. For example, Galileo observations made at a distance of $\sim 60 R_{E}$ from Earth show an ion distribution that appears to be made of three separate bunches [Frank et al., 1994]. To investigate this type of observed nongyrotropic distribution consisting of multiple bunches, a simulation is run using three equidensity bunches of protons symmetrically spaced about the origin. All other parameters are the same as those used in the ring case. Recall that the parameter $\Phi_{0}$ does not depend on the number of bunches, but only the total arc length of the removed azimuthal segment of the nongyrotropic velocity distribution in the perpendicular velocity plane. The example in run 1 therefore has the same value of $\Phi_{0}$, even though the particle bunching is different than the three-bunch case.

[27] The perpendicular velocity space evolution of this distribution is shown in Figure 6 . The main difference between run 1 and run 3 is that in this example, as the three bunches of particles gyrate around the magnetic field in the perpendicular velocity plane, the leading edge (head) and trailing edge (tail) of each bunch of particles diffuses equally in $v_{\perp}$ with no asymmetry. It is interesting that the observations of Frank et al. [1994] follow this same pattern; that is, the two-bunch observation shows the headtail asymmetry, and the distribution with three, nearly symmetric bunches shows little if any asymmetry. Our simulations show similar results. An analytic treatment of the particle dynamics is required to fully understand how this asymmetry is initially formed. The time variation of the four diagnostics for this distribution as well as the growing electric field time histories are essentially the same as in run 1 .

3.2.5. Nongyrotropic distribution: Run 4. [28] Most of the observations of nongyrotropic distributions cited in this text do not show appreciable ion background densities; however, finite background ion populations with gyrotropic Maxwellian distributions are a possibility. To investigate the effect of a gyrotropic background plasma on the nonlinear evolution of the nongyrotropic distribution, we have done several simulations that include a background proton population. Run 4 is one such simulation. Figure 7 shows the total proton distribution in the perpendicular velocity plane for run 4 . The cold proton background is centered at $v_{y}=v_{z}=0$. For this run, $v_{\perp d} / v_{A}=1.0$, 

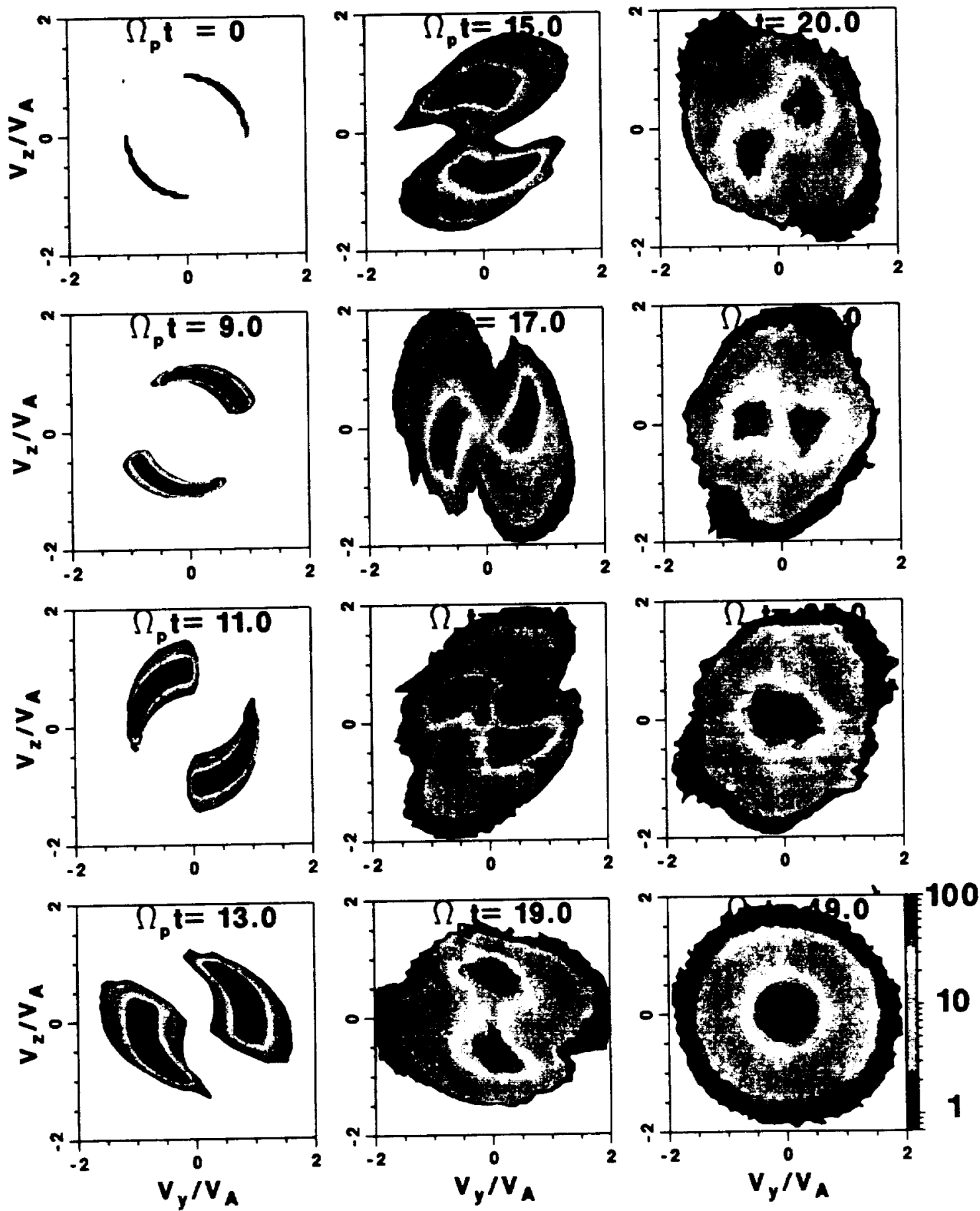

Figure 4. Velociry space time series in the plane perpendicular to the background magnetic field for an initially cold nongyrotropic proton distribution with $\Phi_{\mathrm{o}}=1.0, v_{\mathrm{n}} \mathrm{v}_{\mathrm{A}}=1.0$ and $n_{\mathrm{ng}} / n_{\mathrm{e}}=1.0$

$n_{n \mathrm{~g}} / n_{\mathrm{e}}=0.5$ (i.e. $50 \%$ of the protons make up the background population: $n_{\mathrm{F}}=n_{\mathrm{ng}}+n_{\text {back }}=n_{\mathrm{e}}$ ), and $\Phi_{\mathrm{o}}=1.0$. Throughout the run. background protons (as seen in the perpendicular velocity plane) are diffused preferentially in the direction perpendicular to the leading edge of the nongyrotropic proton distribution. This diffusion toward a Maxwellian-like distribution takes approximately twice as long as a comparable run with no proton background population (i.e., with $\left.n_{\mathrm{ng}}=n_{\mathrm{c}}\right)$. Furthermore, $\left(\delta B / B_{\mathrm{o}}\right)^{2} \max$ is about half the value of the maximum fluctuating field energy of nun 1 . The effect of adding cold background protons to the plasma (and therefore lowering the number of particles in the nongyrotropic distribution) is to reduce the maximum fluctuating field energy in direct proportion to $n_{\mathrm{ng}} / n_{\mathrm{e}}$. The nongyrotropic nature of the distribution remains detectable for a length of time inversely proportional to $n_{\mathrm{ng}} n_{\mathrm{e}}$

\subsection{Parameter Search}

[29] The extent that fluctuating fields produced by nongyro. tropic protons differ from those fields produced by ring protons is now discussed, and the process responsible for saturation of these growing waves is investigated.

3.3.1. Field energy dependence on $\Phi_{0}$. [30] The dependence of the maximum fluctuating field energy on the 

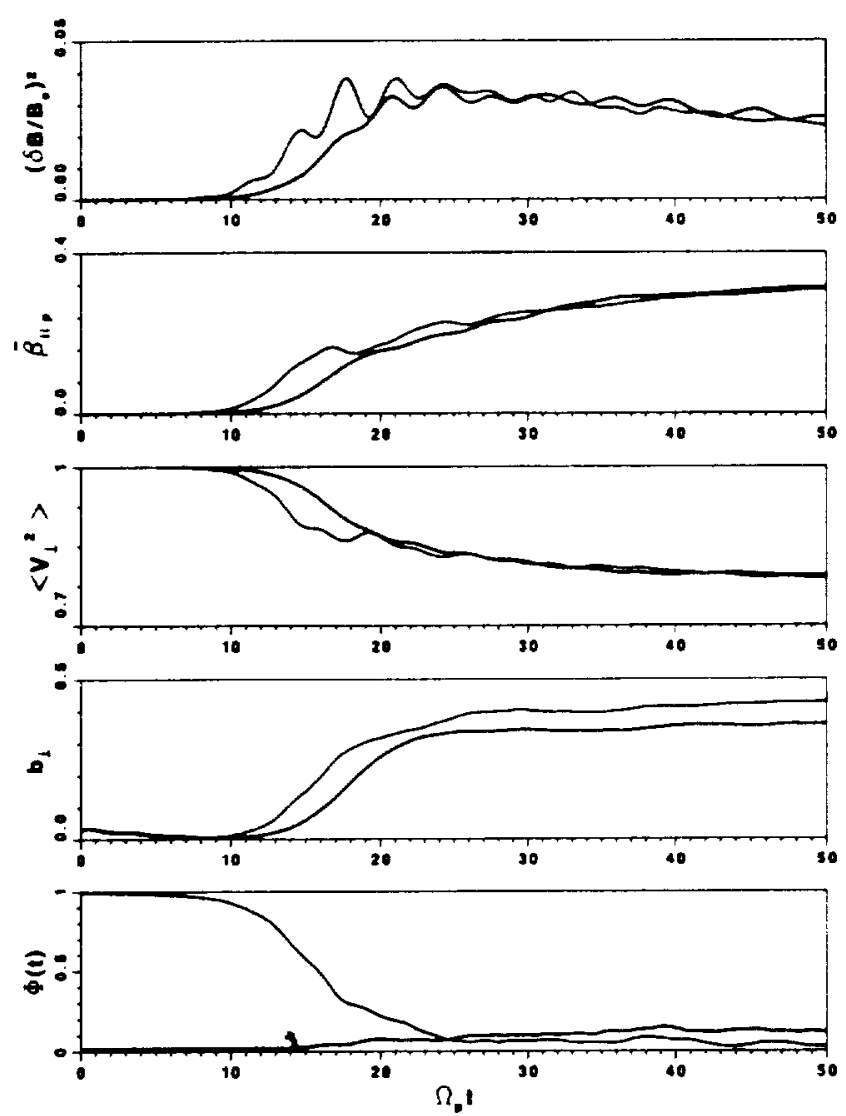

Figure 5. Time evolution of particle diagnostics for simulation run 1 of a nongyrotropic proton distribution, with the same parameters as shown in Figure 4, is shown by the gray line. The ring case (black line) is shown for comparison.

nongyrotropic nature of the protons is shown most clearly in Figure 8. Here $\left(\delta B / B_{0}\right)^{2}$ max is plotted versus $\Phi_{0}$ for $v_{L 0} v_{A}=1.0$ (top panel) and $v_{-\alpha} / v_{A}=2.0$ (bottom panel). In both examples, there are no background protons. As the initial proton distribution is made increasingly nongyrotropic (i.e., increasing $\Phi_{o}$ ) the maximum field energy increases by up to $\sim 60 \%$ in the top panel and by up to $\sim 70 \%$ in the bottom panel over that produced by a gyrotropic ring distribution. This result is significant because it clearly shows that the nongyrotropic nature of the distribution includes a source of free energy not found in the ring distribution.

3.3.2. Field energy dependence on perpendicular beam velocity. [31] Figure 9 shows $\left(\delta B / B_{0}\right)^{2}$ max versus the perpendicular beam speed squared for a series of simulations using either a nongyrotropic distribution (triangles) or a ring distribution (circles) to model the protons. The top panel shows results from runs using nongyrotropic distributions with $\Phi_{\mathrm{o}}=1.0$, while the bottom panel uses $\Phi_{0}=1.5$. Observations of nongyrotropic distributions in the magnetotail have values of $v v_{\mathrm{A}}$ within the range used in Figure 9 . In all runs there are no background protons. The maximum field energy is seen to be an approximate linear function of $\left(v_{-\alpha} v_{A}\right)^{2}$ for the runs using a ning distribution. For runs using a nongyrotropic distribution, this linear relationship holds as well, but only for low values of $v_{\perp 0}$. The nongyrotropic case begins to diverge from the ring case at large perpendicular velocities and larger $\Phi_{0}$, where nongyrotropic effects begin to dominate.

3.3.3. Field energy dependence on nongyrotropic proton density. [32] Figure 10 shows the maximum field energy dependence on density for ring protons (solid line) and nongyrotropic protons (triangles) with $\Phi_{\mathrm{o}}=1.5$ and $v_{-} v_{\mathrm{A}}=10.0$. In Figure 10 a Maxwellian background proton population is used when $n_{\mathrm{ng}} n_{\mathrm{e}}<1.0$. Observations from the magnetotail show that nongyrouropic distributions have low background ion densities, so they would fall toward the right-hand side of Figure 10. The nongyrotropic effects cause the maximum fluctuating energy to diverge sharply from a linear dependence on density for $n_{n y^{\prime}} n_{\mathrm{e}}$ $\geq 0.75$.

3.3.4. Diffusion time for a nongyrotropic distribution to become gyrotropic. [33] Figure 11 summarizes the time it takes for initially nongyrotropic distributions to become gyrotropic. We define the plasma to be gyrotropic when $\Phi(t) \leq 0.1$. and the time it takes for the particles to reach this point is denoted by $i_{i j}$. The top panel shows that $i_{G}$ increases with increasing $\Phi_{0}$. Distributions with larger $\Phi_{1}$, require more diffusion to become gyrotropic, implying a larger $t_{\mathrm{G}}$, but this longer time is partly offset because larger $\Phi_{0}$ also imply larger $\left(\lambda B B_{0}\right)^{2}$ max (as shown in Figure 8) and a corresponding increased diffusion rate of the particles. The bottom panel shows that $t_{0}$ decreases significantly with increasing $v_{\ldots}$. This occurs because as $v_{-}$is increased, fluctuating field amplitudes increase, causing enhanced diffusion.

\subsection{Saturation Mechanism}

[34] The time at which saturation occurs is defined here as the time when fluctuating field energy reaches its maximum value. The simulation results indicate that saturation of the growing tluctuating fields occurs by the same mechanism whether the fields are produced by a ring distribution or by a nongyrotropic distribution. The saturation mechanism is quasi-linear diffusion of the particles leading to an increase in $3_{i \mid p}$ and a decrease in $v_{-}$- until these parameters approach the linear theory threshold values discussed by Convery and Gary [1997] and given by

$$
\frac{v_{\nu}^{2}}{v_{i}^{2}}=\frac{j_{p}}{\left(1-\frac{\lambda_{p}}{\Omega_{p}}\right)}
$$

for $n_{\mathrm{ng}} n_{\mathrm{e}}=1.0$. Saturation was found to be relatively independent of $\Phi_{0}$. The evidence leading to the conclusion that saturation is a quasi-linear process for the nongyrotropic distributions considered here is as follows:

1. If the excited fluctuating field spectrum that causes the diffusion is sufficiently broad in frequency andior wave vector, this process should be adequately described by quasi-linear diffusion [Coroniti, 1985]. The linear theory dispersion for parallel propagating, electromagnetic, proton cyclotron waves in a plasma consisting of Maxwellian electrons and protons modeled by a cold ring distribution produces growing modes that are unstable over a wide range of wavelengths for parameters typically used in our simulations. Our simulation results show enough general similarities between waves produced by ring and nongyrotropic distributions that we might expect similarities in their linear dispersion.

2. In almost all of our simulations, for both ring and nongyrotropic distributions, saturation occurs when both $3_{\| \mathrm{p}}$ and $v^{2}$ are close to the theoretical linear threshold condition given by Conven and Gan [1997]. This implies that quasilinear diffusion, which was found to be the saturation mechanism for waves produced by a proton ring distribution [Convery and Gan, 1997], is also the cause for waves driven by nongyrotropic distributions. Although we have not carried out a linear theory study for a nongyrotropic plasma, we found in the simulations that nongyrotropic distributions saturate at about the same time as the ring simulations and with approximately the same values of $\beta_{l p}$ 

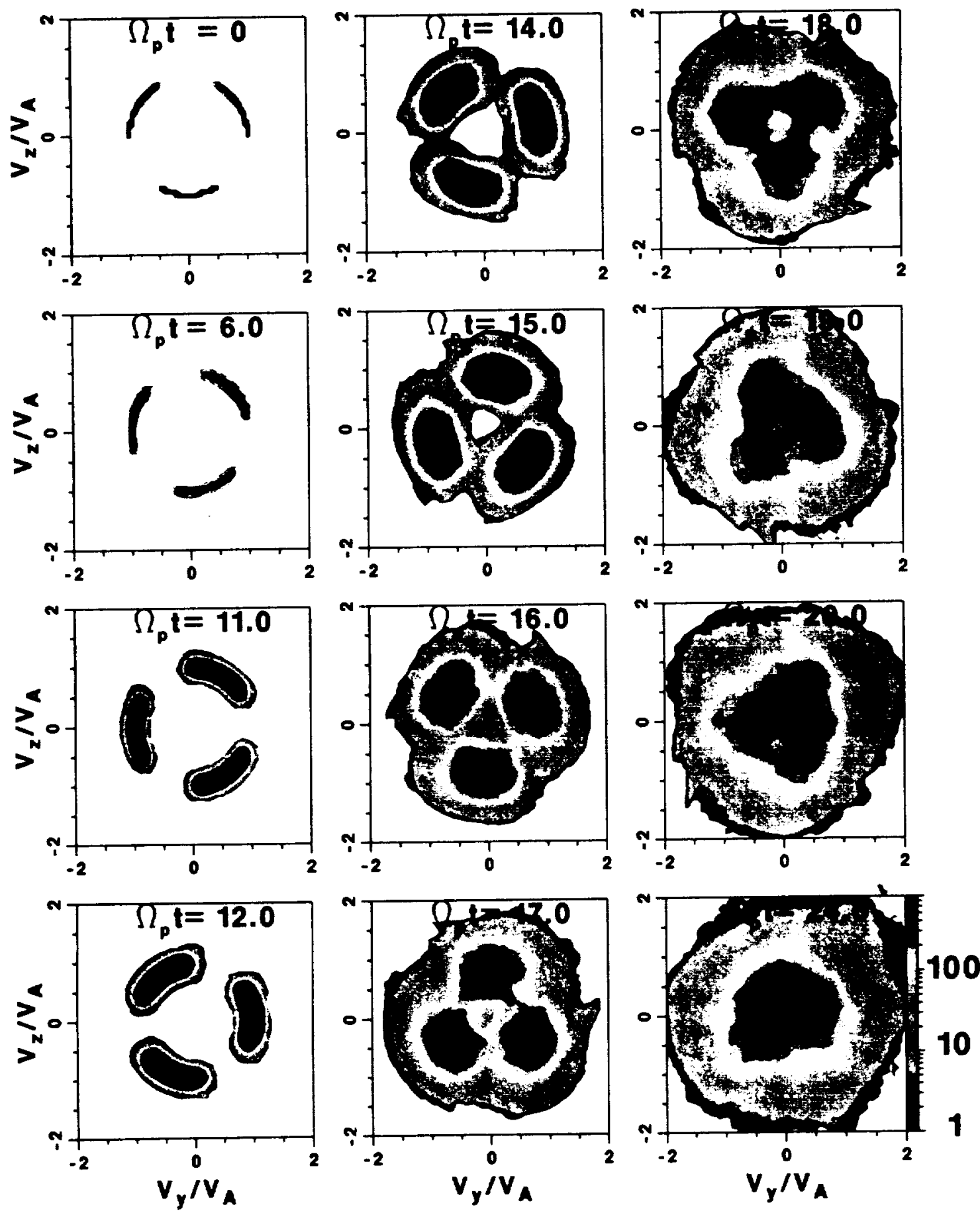

Figure 6. Velocity space time series in the plane perpendicular to the background magnetic field for run 3. which uses an initially cold nongyrotropic proton distribution composed of three symmetrically placed bunches of particles. Here $\Phi_{\mathrm{o}}=1.0,1, v_{\mathrm{A}}=1.0$, and $n_{\mathrm{ng}} n_{\mathrm{e}}=1.0$

and $v_{-}^{2}$. We therefore conclude that quasi-linear diffusion applies in both cases.

3. Another characteristic of quasi-linear diffusion is that the proton distribution should continue to diffuse toward a Maxwellian for some time after saruration. As shown by the velocity space, time history, and particle diagnostic plots discussed previously, the proton distribution diffuses rapidly in $\beta_{\mid i p}$ and $v_{-}{ }^{2}$ up to saturation. The distribution then continues to evolve at a slightly slower rate toward a Maxwellian distribution at late times.

[35] Collisions do not play a role in the saturation process because the Coulomb collision frequency is much lower than the proton gyrofrequency timescale. We rule out particle trapping because the proton distribution continues to diffuse toward a Maxwellian after saturation. If trapping played a role in saturation. the distribution would retain clear non-Maxwellian features for 

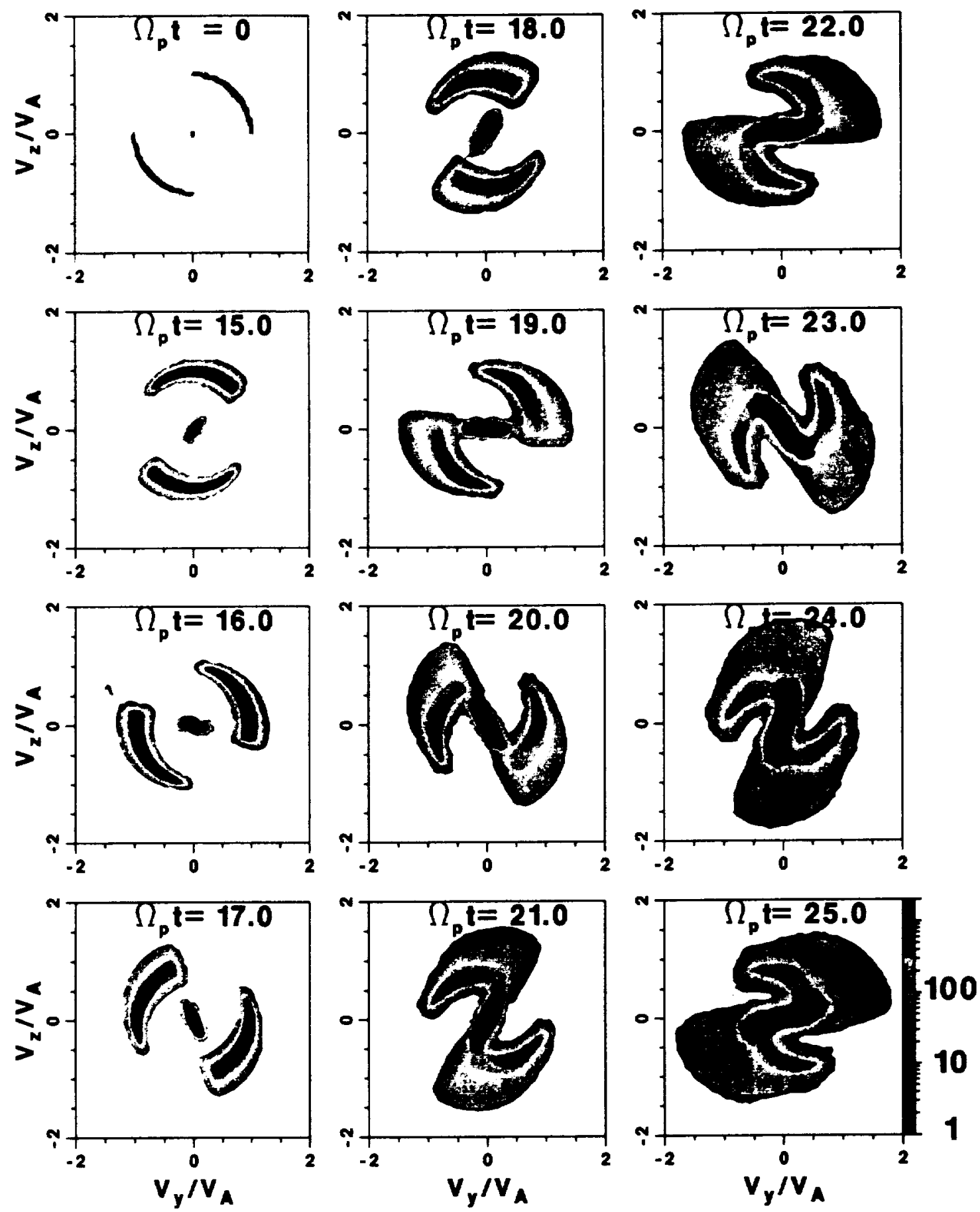

Figure 7. Velocity space time series in the plane perpendicular to the background magnetic field for run 4 , which uses a plasma consisting initially of both cold nongyrotropic protons and background Maxwellian protons. Here $\Phi_{0}=$ 1.0, $v_{-\alpha} v_{A}=1.0$, and $n_{n g} n_{e}=0.5$. Initially, the background particles are preferentially scattered along a line connecting the two leading edges of the nongyrotropic distribution.

some time after saturation. Instead, in our runs, azimuthally bunched features continue to steadily evolve toward a gyrotropic distribution after saturation, with no indication of trapping during late times.

\subsection{Other Characteristics of Nongyrotropic Distributions}

[36] It has been shown that for certain parameter regimes, strongly nongyrotropic distributions can be unstable and generate waves that cause the distributions to quickly diffuse toward a gyrotropic form. This implies that it might be difficult to make direct observations of nongyrotropic distribution functions since most particle instruments require at least several seconds to make a full three-dimensional distribution function measurement. Nevertheless, there are several observations of nongyrotropic distribution functions in the magnetotail and near comets as cited in this paper. Additionally, for the middle and distant magnetotail, there is little or no wave activity detected in the regions where the nongyrotropic distributions are observed. The question arises, if the naturally occurring distributions can be nongyrotropic as the observations 

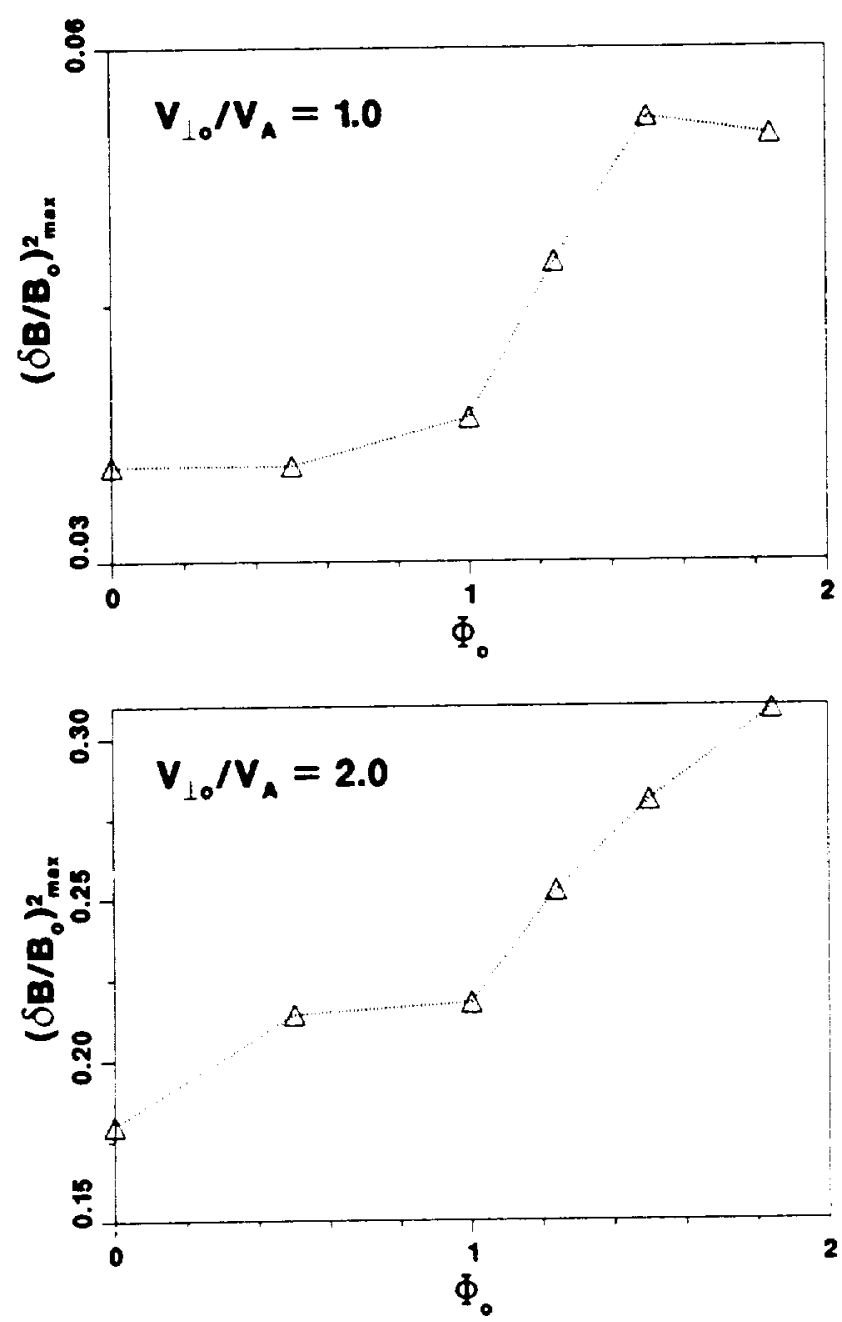

Figure 8. Maximum fluctuating field energy versus $\Phi_{0}$ with (top) $v_{\mathrm{A}}=1.0$ and (bottom) $v_{\mathrm{O}} / v_{\mathrm{A}}=2.0$. In both panels, $n_{\mathrm{ng}} n_{\mathrm{c}}=$ 1.0. Each triangle represents a separate simulation run with a nongyrotropic distribution. The dotted lines connecting the triangles are used simply to clarify the trend of increasing maximum field energy with increasing $\Phi_{\mathrm{o}}$.

suggest. why are they not unstable and diffuse toward a more gyrotropic distribution? We address these imporant questions in this section and show that there are certain parameter regimes in which nongyrotropic distributions are stable. Specifically, we examine the effect of including a finite perpendicular thermal spread to the ring and nongyrotropic distributions. We also investigate single bunched distributions as opposed to the previous sections. where we considered distributions made of two or three symmetrically spaced bunches.

3.5.1. Finite perpendicular thermal spread. [37] Observed nongyrotropic distributions generally show a finite thermal spread in both the parallel and perpendicular velocities, that is. both $T_{\mathrm{i}} \neq 0$ and $T \neq 0$. We have, to this point, used model ring and nongyrotropic distributions that have a finite parallel temperanure but are cold in the perpendicular direction. that is, $T_{-F}=0$. We now include a perpendicular thermal spread in our model nongyrotropic distribution. To do this, the perpendicular velocity $\left(v_{+0}\right)$ of the ring protons is modified by adding a perpendicular thermal speed $v_{\text {th. }}$ such that $T_{-\mathrm{F}}=T_{\mid \mathrm{p} .}$. All examples discussed in this subsection will use $T_{\text {II }}=T_{\perp \mathrm{F}}$. For relatively low perpendicular thermal spreads, the simulation results are very similar to the cold case. As the initial temperature is increased, however, the nongyrotropic distribution eventually becomes stable, independent of the value of $\Phi_{0}$.

[38] The perpendicular velocity space time series for this nongyrotropic case is shown in Figure 12, and the particle diagnostics are given in Figure 13. The effects of using a perpendicular thermal spread in either the initial ring distribution or nongyrotropic distribution, relative to runs using cold initial distributions, are twofold. First, it reduces the growth rate of the instability so that the non-Maxwellian features are more slowly diffused away. that is. $\Omega_{\mathrm{p}} t \approx 40$ for this example compared to $\Omega_{\mathrm{p}} t \approx 25$ for the otherwise identical cold case. Second, the maximum fluctuating field energy is slightly less than in the cold case. Overall, however. the results are qualitatively similar to the cold case.

[39] One similarity that the cold and thermal runs using nongyrotropic distributions have is that in making the distribution more nongyrotropic, $\left(\delta B B_{0}\right)_{\max }^{2}$ is increased over the corresponding gyrotropic ring case. These results provide an answer to the question about the effects of allowing a finite perpendicular temperature in the nongyrotropic distribution. Namely, while warm distributions are a closer representation of the observed distributions, the similarity of our cold and warm results validates our
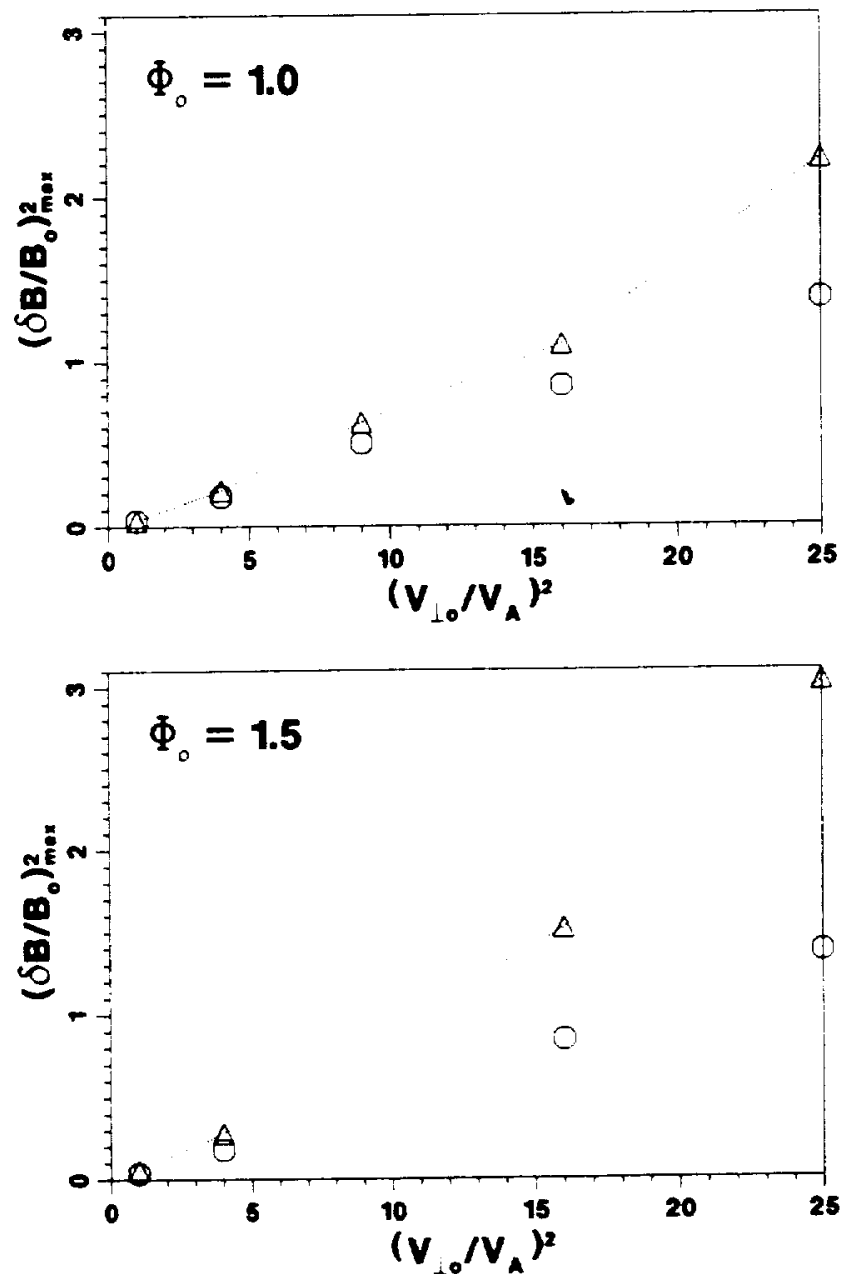

Figure 9. Maximum fluctuating field energy versus perpendicular beam speed squared for a series of simulations using (top) $\Phi_{0}$ $=1.0$ and (bottom) $\Phi_{0}=1.5$. In both panels. $n_{\mathrm{ng}} / n_{\mathrm{c}}=1.0$. Each point represents a separate simulation run. The circles indicate that a ring distribution was used in the simulation, and a triangle denotes that a nongyrotropic distribution was used. All other parameters are the same. 


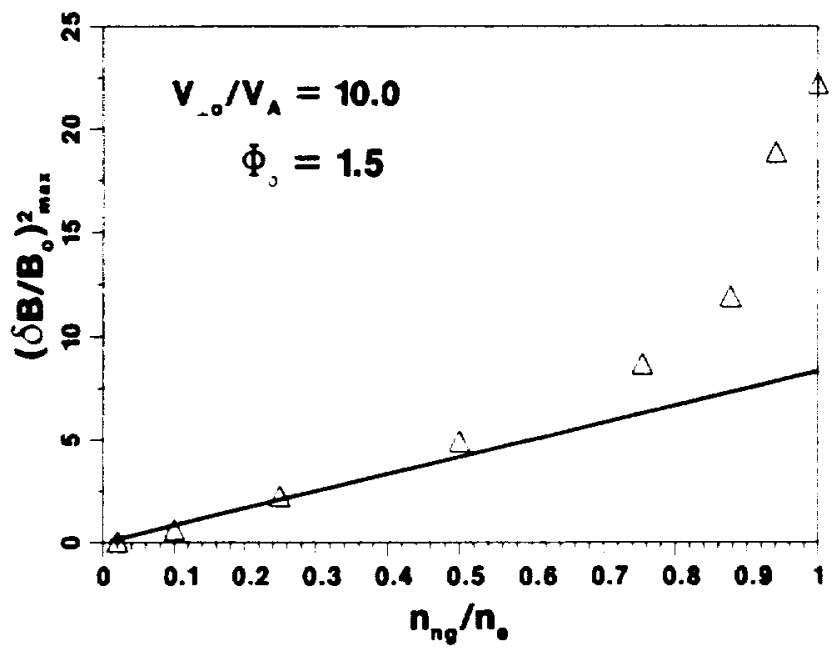

Figure 10. Maximum tluctuating field energy versus $n_{\mathrm{ng}} n_{\mathrm{e}}$ for simulations using $\phi_{0}=1.5$ and $v_{-v} v_{\mathrm{A}}=10.0$. Each triangle represents a separate simulation using a nongyrotropic proton distribution. The solid line is the maximum fluctuating field energy due to an equivalent, cold gyrotropic ring distribution.

initial conclusions gathered from simulations using an initially cold $\left(T_{-p}=0\right)$ model distribution.

[40] Is a distribution function with a finite nongyrotropic nature $\left(\Phi_{0}>0\right)$ a sufficient condition to produce instabilities. or does it simply enhance the field energy of a gyrotropic plasma which is already extant in an unstable parameter regime? This is the second question we seek to answer in this section, that is, is there any parameter regime in which a gyrotropic ring distribution is stable and yet an otherwise identical distribution, but with a nongyrotropic nature $\left(\Phi_{0}>0\right)$, produces an instability?

[41] Our studies show that increasing the initial ring perpendicular thermal spread further produces a decrease in the maximum fluctuating field energy. At a temperature corresponding to $\beta_{\| \mathrm{p}}=$ 0.5 (with $T_{\perp \mathrm{p}}=T_{\mathrm{ip}}$ ), the ring distribution is stable. To examine this, a run was carried out using a nongyrotropic distribution created by removing two $45^{\circ}$ sections $\left(\Phi_{0}=1.5\right)$ from the thermal ring distribution (not shown). The protons in this case did not diffuse significantly until $\Omega_{\mathrm{p}} \approx \approx 50$. The maximum fluctuating field energy reached a peak wave energy that was only $\sim 1 \%$ of the energy produced by an identical run using a cold initial distribution. There was little activity in the other diagnostics except for the slow, gradual decrease in $\Phi(t)$ with time. Additionally, two other runs (not shown) were done using warm nongyrotropic distributions with parameters identical to the first warm nongyrotropic distribution case except for changing $\Phi_{0}$ to 0.5 and 1.0 , respectively. In all three cases the nongyrotropic distribution remained relatively intact with low wave activity, independent of the value of $\Phi_{0}$. These examples imply that $\Phi_{0} \neq 0$ is not a general condition for instability for this parameter regime. It must be linked to the requirement that the nongyrotropic plasma would already be unstable in a related gyrotropic manifestation, at least for thermal parameter values studied here. For unstable distributions, however, $\Phi_{o}$ works well as a diagnostic to encompass the effect of a distribution's nongyrotropic nature on the magnitude of the maximum fluctuating field energy. It is important to point out that in certain parameter regimes other that what is considered here, the introduction of a nongyrotropic plasma distribution by itself can generate an instability when the corresponding gyrotropic siruation is stable. This is true for both stationary [Brinca and Romeiras, 1998; Brinca et al., 1998; Brinca. 1999; Motschmann and Glassmeier, 1998; Motschmann et al., 1999] and time-varying [Motschmann et al., 1999] nongyrotropic distributions.
[42] Two important conclusions can be drawn from these results. First. model distributions with a finite thermal spread in $v_{+}$o give results qualitatively similar to simulations using initially cold perpendicular velocity. However, there are small quantitative differences between the results depending on whether the cold or thermal distribution is used. Therefore the thermal model, which is a more accurate representation of observed nongyrotropic magnetotail plasmas, should be used to make detailed quantitative comparisons with magnetotail observations of quantities such as fluctuating field amplitude and saturation times. Second. for the particular model and limited parameter regime used here. nongyrotropicity (i.e., $\Phi_{0} \neq 0$ ) is not a sufficient condition for the production of tluctuating fields: that is, making a stable ring distribution nongytotropic does not necessarily produce an unstable plasma. These results indicate that the nongyrotropic nature of a plasma population alone does not generate significant fluctuating fields and therefore may be considered stable for some parameters regimes even though the nongyrotropic distribution is non-Maxwellian.

3.5.2. Single bunch distributions. [43] Up to this point our model nongyrotropic distributions have all had one property in common. In the perpendicular velocity plane the nongyrotropic particles are contined in gyrophase in groups of equal number density that are spaced symmetrically about the origin. This model is used because the structure of most hybrid codes. including ours,
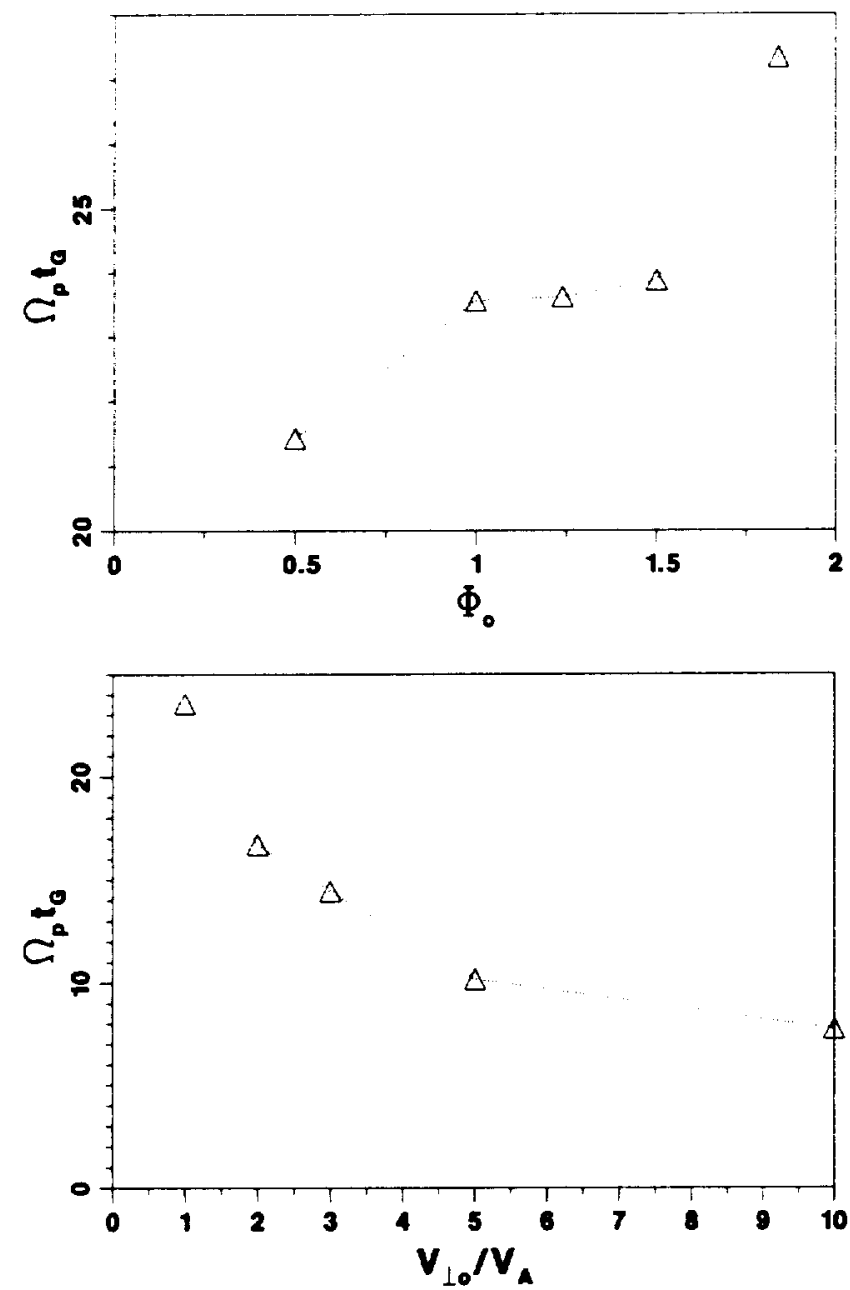

Figure 11. This shows the time for the initially nongyrotropic distribution to become gyrotropic ( $t_{\mathrm{G}}$ ) versus (top) $\Phi_{\mathrm{o}}$ and (bottom) $v_{\perp 0}$, where $t_{\mathrm{G}}$ is defined as the time it takes for the plasma to reach $\Phi(t) \leq 0.1$. 

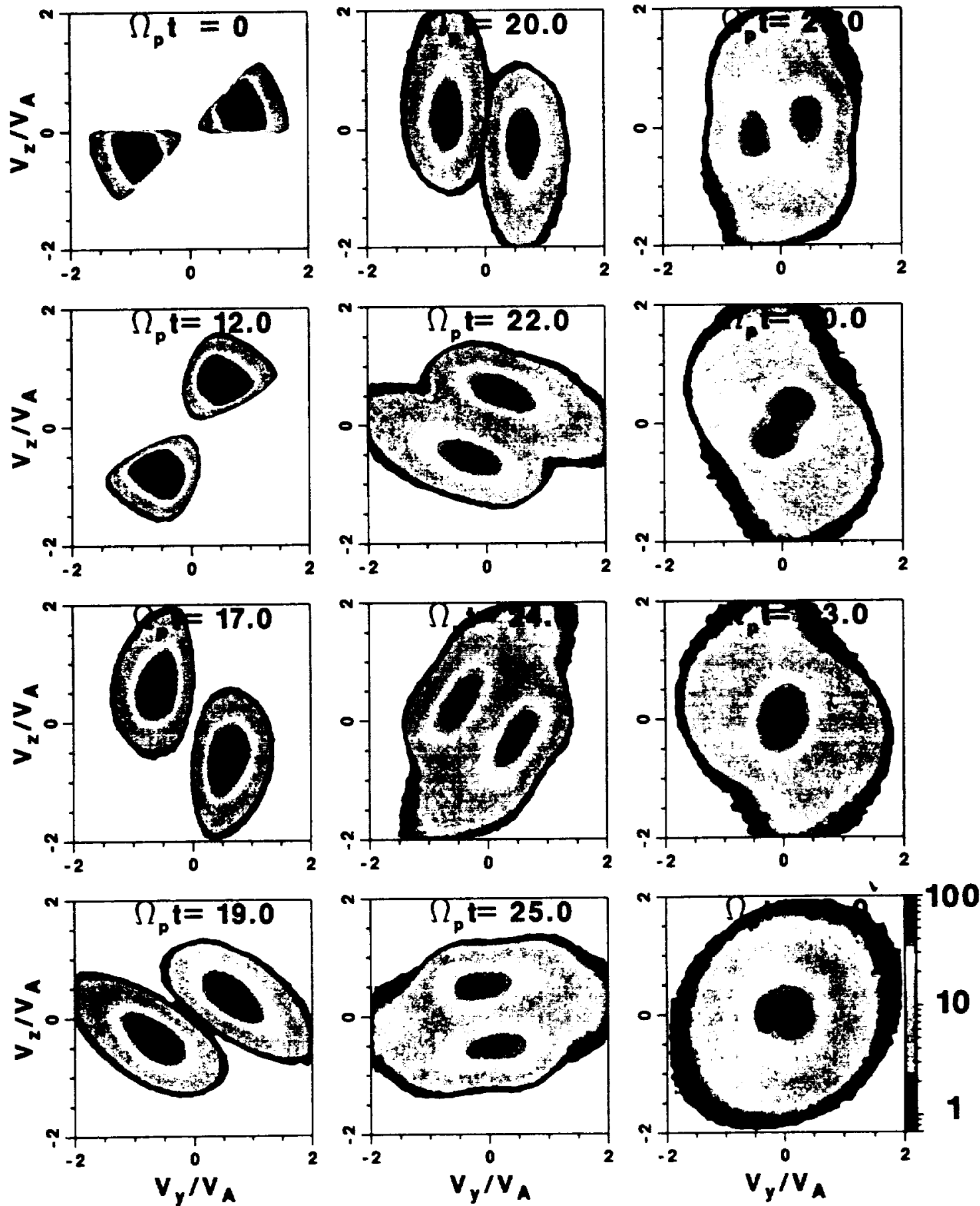

Figure 12. Velocity space time series in the plane perpendicular to the background magnetic field for a nongyrotropic proton distribution with an initial thermal distribution of particles about $v_{-0}$. Here $\Phi_{0}=1.5, v_{-0} \mathrm{v}_{\mathrm{A}}=$ $1.0, n_{\mathrm{ng}} / n_{\mathrm{e}}=1.0$, and $33_{\mathrm{tp}}=0.1$

requires us to assume that there are no net. zeroth order currents While many insightful results have been achieved by using this symmetric, nongyrotropic model. it is worthwhile to explore nonsymmetric distributions because the observed nongyrotropic distributions are typically not symmetrically spaced about the magnetic field. Second. the question arises as to whether the conclusions reached in the analysis of symmetric nongyrotropic distributions apply to nonsymmetric, nongyrotropic distributions as well. Up to now we have parameterized all nongyrotropic effects in a single term. $\Phi_{0}$, that does not take into account the number or the spacing of bunches in a given distribution. only their total azimuthal spread in $\Delta 0$. For example. $\Phi$, has the same value for a distribution made up of two $90^{\circ}$ bunches or a single $180^{\circ}$ bunch. Is $\Phi_{c}$ really a valid way to parameterize the nongyrotropic nature of a distribution? These issues are examined in this section.

[44] Two asymmetric nongyrotropic distributions with different values of $\Phi_{c}$ are studied in this section. Relaxing the requirement of symmetry in perpendicular velocity requires the inclusion of a finite proton background density with an offset velocity set to maintain a net zero initial current Here we are 

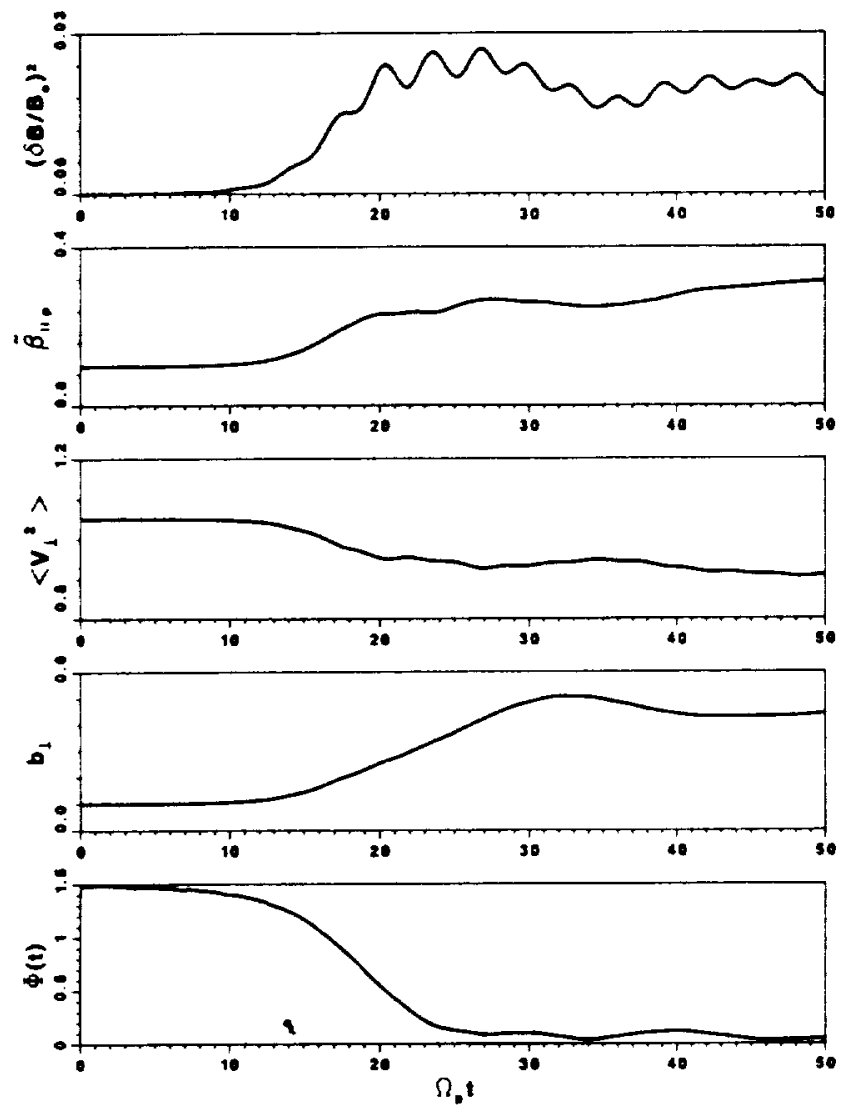

Figure 13. Time evolution of particle diagnostics for the nongyrotropic distribution shown in Figure 12.

concemed mainly with the qualitative aspects of single bunched distributions. In general, larger nongyrotropic densities would most likely produce larger fluctuating field energies for a given value of $v_{\perp o} / \nu_{\mathrm{A}}$. We have been using $v_{\perp \mathrm{o}} / v_{\mathrm{A}}=1.0$ throughout much of this paper; however, we know that a finite background density lowers the maximum field energy (see Figure 10), and we have found that the simulations of this section do not show appreciable growth when using $v_{-\mathrm{o}} / v_{\mathrm{A}}=1.0$. At $v_{-\mathrm{o}} / v_{\mathrm{A}} \approx 2.0$ we begin to see some wave growth; that is, $\left(\delta B / B_{0}\right)_{\max }^{2} \approx 0.01$. For all the nongyrotropic examples considered in this section we use $v_{\perp \mathrm{o}} / v_{\mathrm{A}}=3.0$ and $n_{\mathrm{ng}} / n_{\mathrm{e}}=0.2$, and for comparison, a nun using a cold ring with $n_{\text {ning }} / n_{\mathrm{e}}=0.2$ and $v_{\mathrm{L}} / v_{\mathrm{A}}=3.0$ produced growing field energy with a peak value of $\left(\delta B / B_{0}\right)_{\max }^{2} \approx 0.03$. At saturation $\left(\Omega_{\mathrm{p}} t \approx 20\right)$ the ring distribution was nearly Maxwellian owing to its diffusion in both the parallel and perpendicular velocity.

3.5.2.1. Single bunch nongyrotropic distribution: Example 1: [45] This nun, shown in Figure 14, uses a nongyrotropic distribution created by removing a $90^{\circ}$ section $\left(\Phi_{0}=0.5\right)$ from a cold ring. The field energy reaches a peak value of $\left(\delta B / B_{0}\right)_{\max }^{2} \approx$ 0.035 at $\Omega_{\mathrm{p}} t \approx 16$. Figure 14 shows that the distribution still retains some signs of its initial nongyrotropic character at this time. The main result here is that this single bunch distribution is unstable and produces fluctuating field energy larger than in the ring case.

3.5.2.2. Single bunch nongyrotropic distribution: Example 2: [46] In this example a $270^{\circ}$ section $\left(\Phi_{0}=1.5\right)$ is removed from a cold ring. Figure 15 shows the perpendicular velocity space time history of this distribution. The field energy reaches a peak value of $\left(\delta B / B_{0}\right)_{\max }^{2} \approx 0.06$ at $\Omega_{\mathrm{p}} \mathrm{t} \approx 18$. Thus the maximum field energy, compared to the ring case and to the previous example 1 , increases with increasing $\Phi_{0}$.
[47] From the above examples we conclude that single bunch distributions are similar to the nongyrotropic distributions made of two or more symmetrically spaced bunches that were examined in the earlier sections of this paper. Their characteristics include an increase in maximum field energy with increasing $\Phi_{0}$, and although significant diffusion has taken place in $v$, and $v$, by the time of saturation. the distributions are still nongyrotropic. The main result of this subsection is that the stability of nongyrotropic distributions does not seem to depend on the detailed azimuthal spacing or number of bunches that make up the distribution. This is relevant because nongyrotropic distributions with any number of bunches have been observed in the Eart's magnetotail. We can now have more confidence that the parameter $\Phi_{\omega}$, which does not take into account the number of gyrobunches. gives an accurate description of the maximum tield energy increase due to nongyrotropic effects.

[48] The goal of this section has been to determine which parameters. if any. stabilize nongyrotropic distributions. To this end. we looked at distributions with finite perpendicular thermal spread and those with single bunched distributions. First. we isolated the nongyrotropic nature of the protons by using a distribution characterized only by $\Phi_{0} \neq 0$ (i.e. $\partial f \partial \phi>0$ ) so there was no temperature anisotropy or any other free energy sources, such as ring-like ( $\left.\partial f / \partial \nu_{-}>0\right)$ effects, associated with the plasma. We found that large values of $T_{-p}$ can cause such a nongyrotropic distribution to be stable, even for very large values of $\Phi_{0}$. In this way it is shown that $\Phi_{0} \neq 0$ is not necessarily a sufficient condition for instability in some parameter regimes. It is more accurate to say that if a gyrotropic distribution, such as a ring, is unstable, then nongyrotropic modifications to the plasma (as quantitied by $\Phi_{0}$ ) will enhance the growing fluctuating field energy. In such cases, $\Phi_{0}$ accurately describes the enhanced fluctuating field energy due to the nongyrotropic nature of the plasma. A new parameter that can describe the effect of the nongyrotropic nature of the plasma in all parameter regimes should be developed in the future. This new parameter should be a function of $\Phi_{0}$ and another variable that accounts for the gyrotropic stability of the plasma.

[49] We summarize that nongyrotropic plasmas may be stable if they are characterized by high temperatures in the parallel and/or perpendicular velocity. Other factors contributing to stability are the proton density ratio and the perpendicular beam speed; that is. stability is likely if the nongyrotropic (or ring) distribution density is a sufficiently small fraction of the total plasma density or the particles have small enough values of $v_{-\infty} v_{\mathrm{A}}$ such that linear stability threshold of an otherwise identical gyrotropic ring distribution [Convery and Garv, 1997] cannot be crossed.

\section{Conclusions}

[50] This paper has explored the stability and behavior of nongyrotropic proton distributions. It was motivated by observations from the Geotail [Saito et al., 1994] and Galileo [Frank et al., 1994] spacecraft revealing nongyrotropic distributions in the Earth's magnetotail. These data raised the possibility that standard gyrotropic, Maxwellian-like modeling of particle distributions is an inappropriate description of the plasma in some regions of the Earth's magnetosphere.

[51] Our nongytotropic model distribution has allowed us to deternine consequences of nongyrotropic space plasmas through a comparison with the effects of ring distributions. We conclude that the maximum electromagnetic fluctuating field energy increases as the initial proton distribution is made more nongyrotropic. For the parameters used here, this increase can be as much as 2 times the energy produced by the case using an otherwise identical gyrotropic distribution. The maximum electromagnetic fluctuating field energy for a given $\Phi_{0}$ also increases with increasing $v_{\perp \alpha} / v_{\mathrm{A}}$ and with increasing $n_{\mathrm{ng}} n_{\mathrm{e}}$. The fields stabilize by diffusion in the 

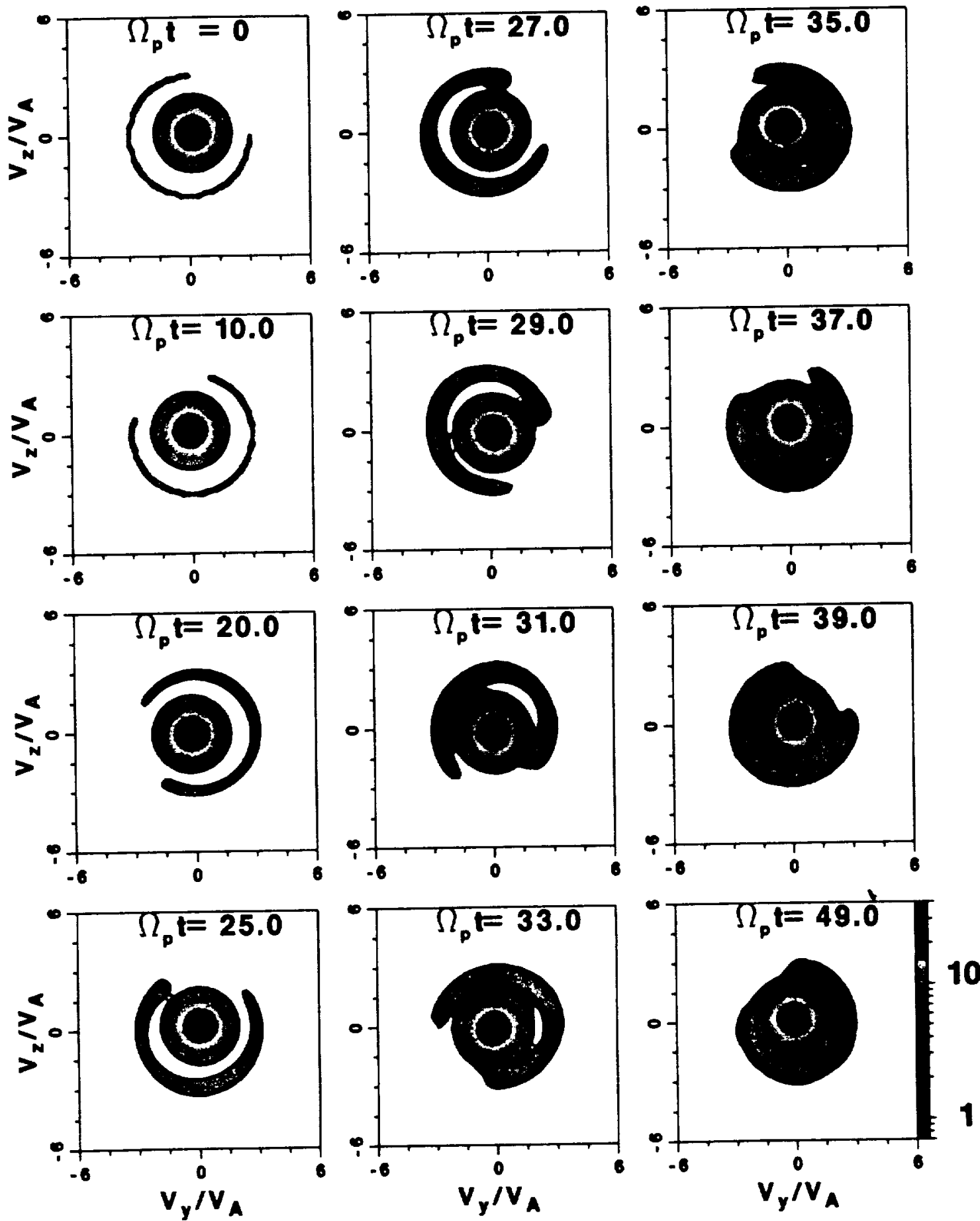

Figure 14. Velocity space time series in the plane perpendicular to the background magnetic field for the case with an asymmetric. nongyrotropic distribution function with a three-quarters ring

parallel and perpendicular velocity. leading to an increase in $\beta_{15}$ and a decrease in $y^{2}$ to values approaching the linear theory instability threshold of Convery and Gan [1997]. The saturation mechanisms and the timing are relatively independent of $\Phi_{0}$. The time for the nongyrotropic distribution to diffuse in phase angle toward a gyrotropic distribution $\left(t_{\mathrm{G}}\right)$ is found to increase with $\Phi_{\mathrm{D}}$ and decrease with $v v_{A}$. This agrees with Cao et al [2000], who showed that pickup ions in solar wind plasmas have increased phase angle diffusion with increased injection velocity.

[52] Cao et al. [1995] and Motschmann and Glassmeier [1993] studied the nongyrotropic ning instability near a cometary environ- ment and found it to be linearly unstable. The linear growth rate of this instability was found to be greatest for a ring distribution and to decrease with an increasing nongyrotropic nature of the distri. bution. The simulation results described in this study show that $\left(c B B_{0}\right)_{\max }^{2}$ increases with the nongyrotropic nature $\left(\Phi_{0}\right)$ of the distribution function, as demonstrated in Figure 8 . In comparing these two results, it should be pointed out that the types of nongyrotropic distributions considered by Cao ef al. [1995] have a different form than the model used here and the parameter regimes of both Cao et al. [1995] and Motschmann and Glassmeier [1993] differ from ours. Thus the linear growth rates cannot 

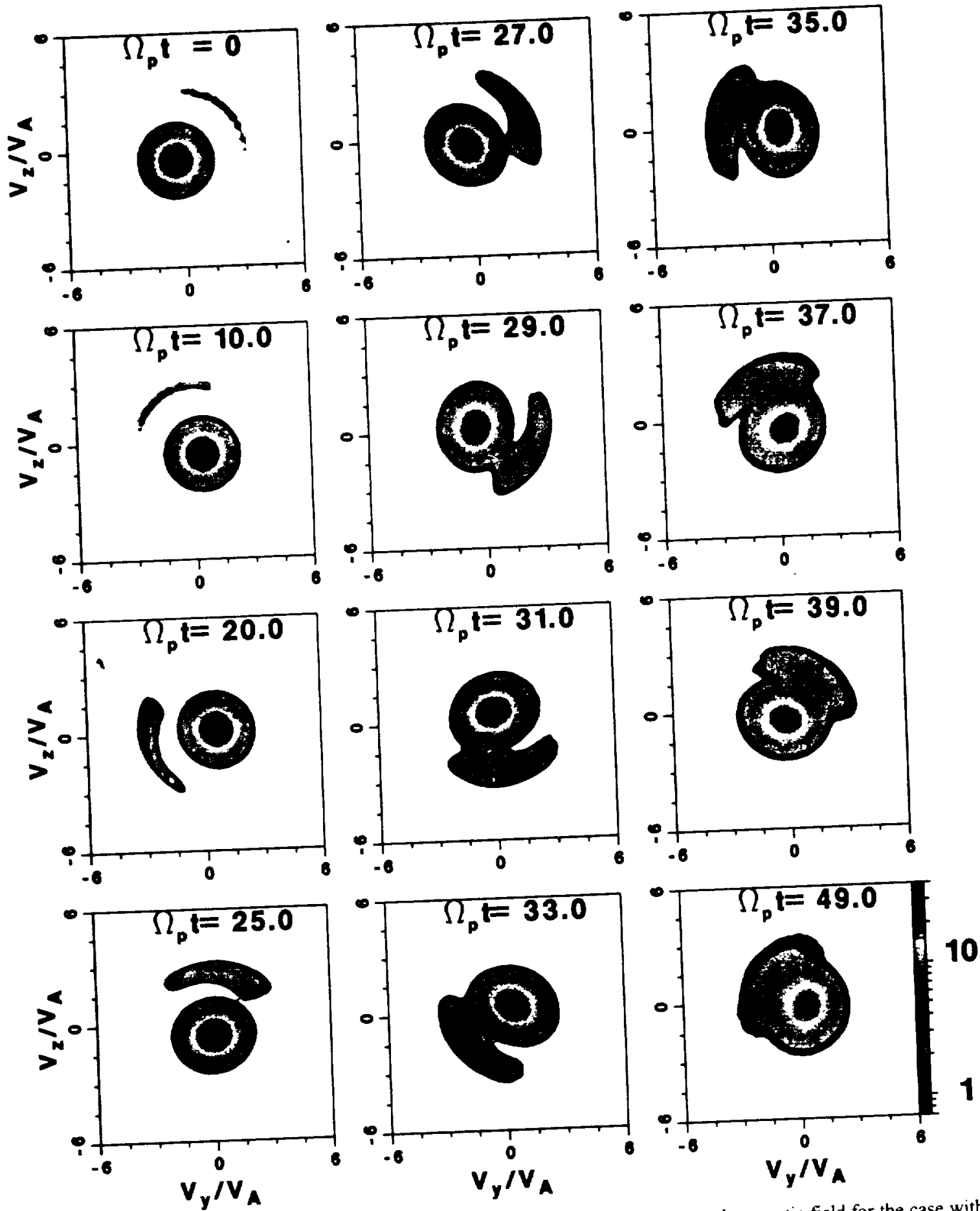

Figure 15. Velocity space time series in the plane perpendicular to the back an asymmetric, nongyrotropic distribution function with a

be easily compared with the results of our computer simulations. parameter regimes it has been demonstrated that the

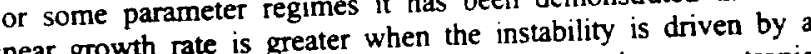

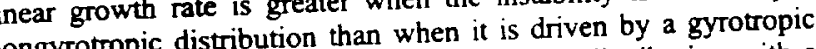
nongyrotropic distribution than whytotropic ion distribution with a temperature anisotropy was found to increase the linear growth rate of parallel propagating electromagnetic waves over the linear row the corresponding gyrotropic ion distrigrown temperature anisotropy [Brinca et al., 1993b] Als an otherwise stable gyrotropic Also, Brinca liven linearly unstable to parallel eigenmodes when a time-varying nongytotropic distribution with a finite perpendicular current is introduced.

[53] It should also be noted that the linear growth rate is not the only determinant of the nonlinear saturation level of fluctuat列 ing field energy described in Figure 8 . For example $\Phi_{0}$ as shown in reach a gyrotropic distribution $\left(t_{\mathrm{G}}\right)$ increases with $\Phi_{\mathrm{o}}$, as shown in Figure 11. This gives waves driven by highly nongyrotropic distributions more time to grow, leading to a larger $\left(\delta B / B_{0}\right)_{\text {max }}$, even if the linear growth rate generated by such a distribution is smaller in comparison to the growth rate produced by a gyrotropic distribution. 
[54] When the gyrotropic plasma is stable, as in the case for a sufficiently warm ring, making the gyrotropic plasma nongyrotropic does not produce instability for the parameters used in this study. When the plasma is in an unstable parameter regime. nongyrotropic distributions produce growing electromagnetic proton cyclotron waves similar to those produced by gyrotropic proton ring distributions, and increasing the nongyrotropic nature (i.e., increasing $\Phi_{0}$ ) will enhance the maximum fluctuating field energy. The growing modes in both the ring and the nongyrotropic cases are parallel propagating and occur at low $\left(\nu_{r} \approx O_{p}\right)$ frequencies. These results lead to the conclusion that the introduction of a nongyrotropic modification to the ring distribution enhances the fluctuating fields that would be produced by a ring distribution. The nongyrotropic nature of a plasma population does not by itself necessarily ensure that the plasma is unstable. at least for the parameters studied here

[55] Similar conclusions were made on the basis of linear stability studies by Brinca and Romeiras [1998] and Brinca [1999], who showed that in certain parameter regimes, parallel propagating waves are stable in the presence of time-varying nongyrotropic distributions, unless a finite perpendicular current exists. Motschmann et al. [1999] found that a time-varying nongyrotropic plasma is linearly stable to parallel propagating waves. for certain parameters, unless an additional energy contribution such as a temperature anisotropy is present. Motschmann et al. [1999] also states that nongyrotropic distributions are expected to be stable when both the perpendicular and parallel velocities are conserved and particle diffusion occurs along the gyrophase angle only. This is because particle energy is independent of phase angle. When the gyrophase diffusion is in conjunction with perpendicular and or parallel velocity diffusion, the particle energy can be converted to wave energy, resulting in growing waves [Motschmann et al., 1999].

[56] A difference found in some of the electric field time histories is that the nongyrotropic distribution is associated with waves having intermediate. fast spikes in amplitude in addition to the low-frequency component near $\dot{\nu}_{1} \approx \Omega_{\mathrm{r}}$. The low-frequency component is similar to that seen in the simulations using a ring distribution. The nongyrotropic distribution produces these waves with little or no linear growth phase. while the ring case shows clear linear growth.

[57] We have discussed observational evidence for the existence of nongyrotropic distributions in the Earth's magnetotail and have established that these distributions lead to enhanced parallel propagating electromagnetic waves. The question that arises now is, what are the magnetospheric consequences of these fluctuating fields? Our simulations show that the growing electromagnetic fields produced by the ring and nongyrotropic distributions saturate by diffusion of the particles in both the parallel and perpendicular directions. The considerable diffusion in the parallel direction could contribute to particle entry into the loss cone, thereby leading to enhanced precipitation. This is significant since particle precipitation calculations that employ the concept of a loss cone traditionally use distributions that are azimuthally symmetric. If fluctuating fields produced by azimuthally bunched distributions change the rate at which particles are scatiered into the loss cone compared to a gytotropic distribution. then it will be necessary for these calculations to be revised for some situations to include nongyrotropic effects. Our simulations indicate that nongyrotropic particles are associated with fluctuating fields having enhanced wave energy above that of the gyrotropic case. This leads to an increase in the diffusion rate over the gyrotropic case

[58] If field lines in the outer boundary of the magnetotail plasma sheet are connected to the auroral oval, this indicates that particles lost in the way described above could contribute to the aurora and low-altitude ion flux coming from the tail. This loss mechanism is significant because il affects particles in the central plasma sheet that otherwise would be unlikely to have $v$ large enough to enter the loss cone. For example. large-scale kinetic particle trajectory studies show' that in the absence of wave-particle interactions, only a small fraction of plasma sheet boundary layer ions have pitch angles that are small enough to be able to precipitate into the auroral ionosphere [Ashour-Abdalla et al.. 1993a]. There have been other suggestions for maintaining ion precipitation from this region. For example. Zelenyi et al. [1990] suggested that stochasticity could lead to an effective diffusion in the CPS resulting from particles undergoing many crossings of the field reversal region and their subsequent acceleration. Neither this mechanism nor the process discussed here of increased ion precipitation due to instabilities associated with nongyrotropic distributions rules out the other. These processes both involve acceleration in the magnetotail field reversal region. and they may be related or may be operating simultaneously to effect the observed ion precipitation. The results here indicate that the effects of waves in the presence of nongyrotropic distribution functions must be considered self-consistently to fully understand ion precipitation from the magnetotail.

[59] Acknowledgments. The authors would like to thank S. P. Gar. and $M$. El-Alaoui for useful comments and discussion related to this project. This research was funded by NASA ISTP grant NAG5-6689 and NASA grant NAG5-8085. Computing resources were provided by the NSF National Partnership for Advanced Computing Infrastructure (NPACI)

[60] Janet G. Luhmann thanks Jinbin Can and Christian Mazelle for their assistance in evaluating this paper.

\section{References}

Ashour-Abdalla. M.. J. Berchem. J. Büchner, and L. M. Zelenyi. Shaping of the magnetotail from the mantle: Global and local structuring. $J$. Georphis. Res., 98, 5651, 1993a.

Ashour-Abdalla M., L. M. Zelenyi. V. Peroomian. and R. L. Richard. On the structure of the magnetotail curent sheet. Geophis Res Lett. 20 . 2019. 1993b.

Ashour-Abdalla. M.. L. M. Zelenyi. V. Peroomian. and R. L. Richard. Consequences of magnetotail ion dynamics. J Geophys. Res. 99. 14.891. 1994.

Ashour-Abdalla. M.. L. A Frank. W: R Paterson. V. Peroomian. and L. M. Zelenyi. Proton velocity distributions in the magnetotail: Theory and observations.J Geophis. Re's.. 101. 2587. 1996

Astudillo. H. F.. S. Livi. E. Marsch. and H. Rosenbauer. Evidence for nongyrotropic aipha particle and proton distribution functions: TAUS solar u'ind measurements.J Geophirs Res.. 101. 24.423. 1996.

Bnnca. A. L. Stability of stationary and time-varying nongyrotropic particle distributions. Ann. Geophys. 1-, 623, 1999

Bnnca. A. L.. and F J. Romeiras. On the stability of stationan nongyrotropic distribution functions: Coupling and purely growing waves. J. Geophis Res. 103.9275, 1998

Brinca. A. L.. L. Borda Aqua. and D. Winske. Nongytotrop as a source of instabiity and mode coupling. Geophrs Res. Let7. 19. 2445.1992.

Brnca. A L.. Y. Omura. and H. Matsumoto. Linear coupling effects onginated in electron nongyrotropy. J Geophis Res.. 98.21.071. 1993a.

Brinca. A. L., L. Borda Agua. and D. Winske. On the stability of nongyrotropic ion populations. A first (analyuc and simulation) assessment. $J$ Geophis. Res. 98. 7549. 1993b.

Brnca. A. L.. Y. Omura. and H. Matsumoto. Stability of perpendicular propagation in time-varying nongyrotropic plasmas: Simulations. $J$ Getphis Res. J03. 29.493.1998

Burnskaya. T. D Schriver. and M. Ashour-Abdalia. Cieneration of electrostatic waves as a consequence of chaotic ion acceleration in the neutral sheet. Geophis. Res. Letl. 21. 2083. 1994

Cao. J. B.. C. Mazelle. G. Belmont. and H Reme. Nongyrotrop: of heary newborn ions at comet Grigg-Skjellerup and corresponding instability. J. Geophus Res.. J00. 23.379, 1995

Cao. J. B. C. Mazelle. G Beimont and H Reme. Oblique ring instability driven by nongyrotropic ions: Application to observations at comet Grige-Skiellerup. J Geophys Res. I03.2055. 1998

Cao. J. B.. C. Mazelle. and G. C Zhou. Phase angle diffusion of newborn ions in the self-consistently generated fields in solar w'ind plasmas. Phys Plasmas. 7(2), 696, 2000

Coates. A. J.. A. D Johnstone. B. Wilken, and F. M Neubauer. Velociry space diffusion and nongyrotrops of pickup water group lons at comel Grigg-Skiellerup. J Geophy.s. Res. 98. 20.985. 199? 
Convery, P. D., and S. P. Gary, Electromagnetic proton cyclotron ning instability: Threshold and saturation, J. Geophys. Res., 102, 2351, 1997.

Coroniti. F. V., Space plasma turbulent dissipation: Reality or myth?, Space Sci. Rev. 42, 399, 1985

Delcourt, D. C. and R. F. Martin. Jr., Application of the centrifugal impulse model to particle motion in the near-Earth magnetotail, $J$. Geophys. Res., $99,25.583,1994$.

Delcourt, D. C., and R. F. Martin, Jr., Pitch angle scattering near energy resonances in the geomagnetic tail, $J$ Geophys. Res.. 104. 383. 1999.

Eastman. T. E.. R. R. Anderson. L. A. Frank, and G. K. Parks, Upstream particles observed in the Earth's foreshock region. $J$ Geophys. Res., 86. $4379,1981$.

Frank. L. A., W. R. Paterson, and M. G. Kuvelson, Observations of nonadiabatic acceleration of ions in the Earth's magnetotail. J. Geophys. Res. $99,14.877,1994$

Fredericks. R. W., A model for generation of bow-shock-associated upstrearn waves. $J$. Geophys. Res. 80. T. 1975

Freund, H. P., J. Q. Dong, C. S. Wu. and L. C. Lee, A syclotron-maser instability associated with a nongyrotropic distribution. Phys. Fluids, 30, 3106. 1987.

Fruchtman. A., and L. Friedland. Theory of a nonwiggler collective free electron laser in uniform magnetic tield, IEEE J. Quantum Electron., $Q E$ 19,1983

Gurgiolo, C., and H. K. Wong, Low and high frequency waves generated by gytophase bunched ions at oblique shocks. Geophys. Res. Lett., 20, 783, 1993.

Motschmann, U. M., and K. H. Glassmeier, Nongyrotropic distribution of pickup ion at comet $P$ /Grigg-Skellerup: A possible source of wave activity, J. Geophys. Res. 98, 20,977, 1993

Motschmann, U. M., and K. H. Glassmeier, Dispersion and wave excitation in nongyrotropic plasmas. $J$. Plasma Phys., 60. 1!1, 1998
Motschmann. U. M.. H. Kafemann, and M. Scholer. Nongyrotropy in magnetoplasmas: Simulation of wave excitation and phase space diffusion. Ann. Geophys., 15, 603, 1997.

Motschmann, U. M., K. H. Glassmeier, and A. L. Brinca, Nongyrotropic particle distributions in space plasmas, Ann. Geophvs., /7. 613, 1999.

Neubauer, F. M. K. H. Glassmeier. A. J. Coates, and A. D. Johnstone. Low-frequency electromagnetic plasma waves at comet $\mathrm{P}$ Grigg-Skjellerup: Analysis and interpretation, $J$ Geophys. Res.. 98. 20.937. 1993

Saito, Y. T. Mukal, M. Hirahara. S. Machida A. Nishida, T. Terasawa. S. Kokubun and T. Yamamoto. GEOTAIL observation of ning-shaped ion distribution functions in the plasma sheet-lobe boundary. Geophys. Res. Lett. 2I. 2999.1994.

Sudan. R. N.. Growing waves in a nongyrotropic plasma. Phys. Fluids, 8, 1965,1915

Winske, D and $N$. Omidi. Hybrid codes: Methods and applications, in Computer Space Plasma Phvsics: Simulation Techniques und Sottware, edited by H. Matsumoto and Y. Omura. pp. $103-160$. Terra Sci., Tokyo. 1993

Zelenyi, L.. A. Galeev, and C. F. Kennel, Ion precipitation from the inner plasma sheet due to stochastic diffusion. J. Geophys. Res. 95. 3871, 1990

M. Ashour-Abdalla and P. D. Convery, Department of Physics and Astronomy. University of Califomia at Los Angeles. Los Angeles, CA 90095. USA.

R. L. Richard and D. Schriver, Institute of Geophysics and Planetary Physics. University of Califomia at Los Angeles. 3860 Slichter Hall, 405 Hilgard Avenue. Los Angeles. CA 90095-1567. LSA. (dave $a$ igpp ucla.edu) 